Elsevier Editorial System(tm) for

Communications in Nonlinear Science and Numerical Simulation

Manuscript Draft

Manuscript Number: CNSNS-D-19-00560R1

Title: A novel method based on augmented Markov vector process for the time-variant extreme value distribution of stochastic dynamical systems enforced by Poisson white noise

Article Type: Research Paper

Section/Category: Modeling, analysis and simulations(ruffo)

Keywords: Time-variant extreme value process; Augmented Markov vector process; Stochastic dynamic system; Poisson white noise excitation

Corresponding Author: Dr. Jianbing Chen,

Corresponding Author's Institution: Tongji University

First Author: Meng-Ze Lyu

Order of Authors: Meng-Ze Lyu; Jianbing Chen; Antonina Pirrotta, Ph.D.

Abstract: The probability density function (PDF) of the time-variant extreme value process for structural responses is of great importance. Poisson white noise excitation occurs widely in practical engineering problems. The extreme value distribution of the response of systems excited by Poisson white noise processes is still not yet readily available. For this purpose, in the present paper, a novel method based on the augmented Markov vector process for the PDF of the time-variant extreme value process for a Poisson white noise driven dynamical system is proposed. Specifically, the augmented Markov vector (AMV) process is constructed by combining the extreme value process and its underlying response process. Then the joint probability density of the AMV can be evaluated by solving the Chapman-Kolmogorov Equation, e.g., via the path integral solution (PIS). Further, the PDF of the time-variant extreme value process is obtained, and can be used, say, to estimate the dynamic reliability of a stochastic system. For the purpose of illustration and verification, several numerical examples are studied and compared with Monte Carlo solution. Problems to be further studied are also discussed. 
Highlight

- The time-variant probability density function of extreme value of a system driven by Poisson white noise is studied.

- An augmented Markov vector process is constructed by combining the state vector and the extreme value process.

- A path integral solution with high efficiency is proposed.

- Several examples are illustrated, demonstrating the effectiveness of the proposed method. 

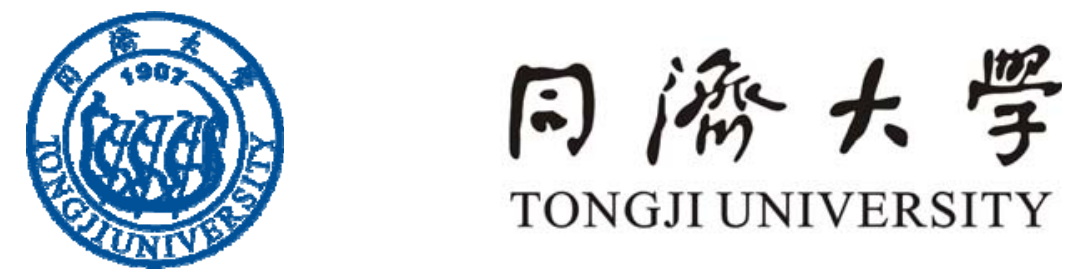

June 19, 2019

Dear Editor,

Thank you very much for your email dated on 26 May 2019 attaching the reviewing comments. We studied the reviewing comments carefully, and made revisions on the manuscript accordingly. Attached please find the revised manuscript titled "A novel method based on augmented Markov vector process for the time-variant extreme value distribution of stochastic dynamical systems enforced by Poisson white noise" re-submitted to the journal Communications in Nonlinear Science and Numerical Simulation for possible publication.

Attached also please find the separate file of statements of revisions. For clarity, the manuscript was revised in a format of modification tracing.

If there are any problems, please be kind to let me know. Thank you very much for your kind help. We are looking forward to the reviewing comments.

Best regards,

Yours sincerely

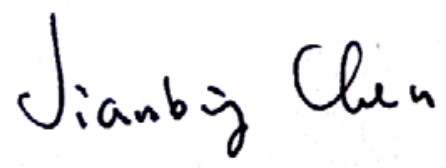

Jianbing Chen

Ph.D., Professor

School of Civil Engineering

\& State Key Laboratory of Disaster Reduction in Civil Engineering

Tongji University

1239 Siping Road, Shanghai 200092, P.R.China

E-mail: chenjb@tongji.edu.cn 


\section{Statement on the Revisions and Responses to Reviewing Comments}

The reviewing comments have been carefully studied. They are very constructive and helpful for us to revise and improve our paper. Accordingly, the revisions were made. To be clear, the manuscript was revised in the format of modification tracing. The main revisions in the paper and the point-to-point response to the reviewing comments are as follows:

\section{Responses to Reviewer \#1:}

1.1. Comments: The authors present a new method for computing the extreme value response of systems excited by Poisson white noise. The computation of the time-varying extreme value response is a challenging task and not many methods are available to deal with this this problem.

The method presented by the authors consists in forming an augmented Markov vector process by combining the extreme value process and the system state. The joint pdf of this vector is computed by path integration. These are rather standard techniques, however applied to a new and challenging problem, and they are limited to low dimensional state vectors.

In summary, the paper is acceptable and adequate for the journal, but there are several minor corrections that have to be carried out. Most of them concern language and grammar issues.

Response: Thanks a lot for the positive comments.

1.2. Comments: Page 2: "trucks" instead of "tracks", "airplane" instead of "airplanes", "the response" instead of "response", Kolmogorov equations" instead of "Kolmogorov equation", "numerical methods" instead of "the numerical methods", "are available in" instead of "available". "Such a treatment is inconvenient": Give a justification!

Response: Thanks for the reminding. The language expression has been modified accordingly. A justification of the drawback of absorbing boundary condition method has been given in the end of the $2^{\text {nd }}$ paragraph of Sec. 1 . 
1.3. Comments: Page 3: "errors" instead of "error", "is then elaborated" instead of "are then elaborated".

Response: Thanks for the reminding. The language expression has been modified accordingly.

1.4. Comments: Page 4: "Heaviside" instead of "Heaviside's".

Response: Thanks for the reminding. The language expression has been modified in the paper.

1.5. Comments: Examples: Please indicate the deviation of the Poisson excitation from a Gaussian white noise excitation and also that of the response and the extreme values from those obtained by a comparable Gaussian excitation. Justify the choice of the values for lambda.

Response: To make it clear, the difference between Gaussian white noise excitation and Poisson excitation and the response are visualized by showing two typical samples of the Poisson excitation in the newly added Fig. 13 in Example 4. Because the Gaussian white noise is well known, it is not shown here. It is seen clearly from Fig. 13 that in the compound Poisson process are stepwise and thus the Poisson white noise is discrete spikes, which is quite different from the white noise. For Poisson white noise excitation, the rate $\lambda$ is a finite value, such as $\lambda=3$ which is taken in the examples in the paper. When the rate $\lambda \rightarrow \infty$, and $\lambda \tau \rightarrow 1$, the Poisson white noise excitation becomes Gaussian white noise.

1.6. Comments: Conclusion: Please rewrite the following statement "programming can be processed to have higher numerical storage and computational efficiency".

Response: Thanks for the reminding. The Ref.[36] has been added behind the Sec.4. (Chen JB, Lyu MZ. A new approach for the time-variant probability density function of the maximum value of a Markov process. Physica A, 2019 (under review).) The numerical technology has also been mentioned in the $2^{\text {nd }}$ last paragraph of Sec.4 and has been elaborated in detail in Ref.[36]. Therefore, the numerical technology will not be repeated to avoid lengthiness.

Again, thank you for your positive and constructive comments, especially for the details to be corrected. 


\section{Responses to Reviewer \#2:}

2.1. Comments: The present paper investigates the evaluation of the probability density function (PDF) of the time-variant extreme value process for a dynamical system driven by Poisson White Noise.

The authors propose a method based on the "augmented Markov vector" (AMV) process for the PDF which combines the extreme value process and its underlying response process.

The Path Integral Solution is used to evaluate the joint probability density of the AMV.

The topic of the paper is interesting and the presented results show the efficiency of the numerical procedure; however some comments arise.

Response: Thanks a lot for the positive comments.

2.2. Comments: In the reviewer opinion the section 2 related to the Poisson White Noise excitation is recurring and not so clear. In particular:

- Eq.5 is not clear; some more explanatory comments have to be added.

- The definition of $<$ DELTA $>W P(t)$ is reported before Eq.5 and after Eq.8.

Response: Thanks for the reminding. Eq.(5) has been modified, and more explanatory has been added after Eq.(5). The definition of $\Delta W_{\mathrm{P}}(t)$ after Eq.(8) has been deleted.

2.3. Comments: The sentence "monotonous in the sense of sample" in section 3 is not clear.

Response: Thanks for the reminding. More explanatory has been added after Eq.(10).

2.4. Comments: Some more comments concerning the augmented vector process and its property of Markovian process could be added.

Response: Thanks for the reminding. More explanatory has been added in the last paragraph of Sec.3.

2.5. Comments: An effort with some more comments on the cited procedure to implement the 
PIS could be help the reader.

Response: Thanks for the reminding. The numerical technology has been mentioned in the $2^{\text {nd }}$ last paragraph of Sec.4 and has been elaborated in detail in Ref.[36]. (Chen JB, Lyu MZ. A new approach for the time-variant probability density function of the maximum value of a Markov process. Physica A, 2019 (under review).) Therefore, the numerical technology has not been repeated to avoid lengthiness. For clarity, we added the reference.

2.6. Comments: Several numerical examples with different distributions for the Compound Poisson Noise are considered and results are reported for different expressions provided for the transition probability density. The section 5 appears again repetitive. In all the reported figures the axis label is "P" which maybe stands for "PDF" Is it correct? However some graphics show the CDF.

Response: Thanks for the reminding. In all the reported figures, the axis label ' $P$ ' stands for 'probability', and ' $p$ ' for 'probability density'. Considering that the two symbols are easily confusing, the axis label ' $\mathrm{CDF}$ ' is used to stand for 'probability', and 'PDF' for 'probability density' in all the figures of the modified version.

2.7. Comments: Moreover I suggest that the authors have to read carefully the final version of the manuscript in order to always have grammatically correct English. Many errors and misprints are present.

Response: Thanks for the reminding. The grammar and spelling errors in the paper has been modified furtherly.

2.8. Comments: On the understanding that the authors will elaborate the manuscript complying with the arised points, the paper could be considered for publication in Communications in Nonlinear Science and numerical simulations.

Response: Thanks a lot for the positive comments.

We appreciate your constructive and kind suggestions which help us to improve our statements greatly. 


\section{Responses to Reviewer \#3:}

3.1. Comments: The extreme value distribution is very important in various science and engineering fields, especially for the systems driven by Poisson white noise. However, it is very difficult to obtain such information either by analytical or numerical methods. In the present manuscript, a novel method was proposed. The basic idea is to introduce an auxiliary extreme value process, and then an augmented vector process combining the extreme value process and the state variables was constructed and proved to be Markov. The Chapman-Kolmogorov equation was thus advocated and facilitated by the path integral solution. Several examples were exemplified in detail, showing the effectiveness of the proposed method by comparing with the Monte Carlo simulation. In particular, the jumps/discontinuity in PDF of extreme value can be captured.

Response: Thanks a lot for the positive comments.

3.2. Comments: In the third line of the second paragraph of the Introduction, the author mentioned that "It is difficult to capture the analytical solution of its PDF, but the numerical results can be obtained by solving the Kolmogorov-Feller equation or the corresponding integral equation, Chapman-Kolmogorov equation using the numerical method, e.g. the finite element method $\cdots . . .$. ". Here, I would suggest the update the literature to include J. Appl. Mech 84(9), 091004 (Jul 12, 2017) , doi: 10.1115/1.4037158; J of Comp Phys (2019), https://doi.org/10.1016/j.jcp.2019.05.023.

Response: Thanks for the reminding. Two suggested references have been added in Sec.1 as Refs.[7] and [15]. These references are very valuable in this field, and closely relevant to the present paper.

3.3. Comments: The manuscript is on the whole well organized with innovations. The reviewer believe it will be a valuable contribution to the literature and can be accepted for publication.

Response: Thanks a lot for the positive comments.

Great thanks to you for your valuable suggestions that make our paper more completed. 
Taking this opportunity, we also smooth the manuscript thoroughly once again. In the modified version, all the figures have been regenerated, and $10^{6}$ samples in Monte-Carlo simulation have been taken for each example to compare with the proposed method in more detailed in the tail of PDF.

In summary, encouraged by reviewers and distinguished editor to revise our previous manuscript, we did our best to improve it and completed some changes in the revision. These changes improve our paper greatly. All responses on the reviewing comments are listed above after our careful discussions. We hope the revisions and responses meet the reviewing comments. Once again, thank the reviewers very much for the constructive comments and suggestions. 


\title{
A novel method based on augmented Markov vector process for the time-variant extreme value distribution of stochastic dynamical systems enforced by Poisson white noise
}

Meng-Ze Lyu ${ }^{\text {a }}$, Jian-Bing Chen ${ }^{\mathrm{a},{ }^{*}, \text { Antonina Pirrotta }}{ }^{\mathrm{b}, \mathrm{c}}$

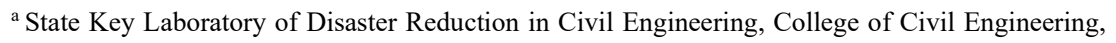
Tongji University. Siping Road, Shanghai 200092, P. R. China

${ }^{b}$ Dipartimento di Ingegneria Civile, Ambientale Aerospaziale e dei Materiali (DICAM), Università degli Studi di Palermo. Viale delle Scienze, Palermo 90128, Italy

${ }^{\mathrm{c}}$ Department of Mathematical Sciences, University of Liverpool. Liverpool L69 7ZL, UK

\begin{abstract}
The probability density function (PDF) of the time-variant extreme value process for structural responses is of great importance. Poisson white noise excitation occurs widely in practical engineering problems. The extreme value distribution of the response of systems excited by Poisson white noise processes is still not yet readily available. For this purpose, in the present paper, a novel method based on the augmented Markov vector process for the PDF of the time-variant extreme value process for a Poisson white noise driven dynamical systems is proposed. Specifically, the augmented Markov vector (AMV) process is constructed by combining the extreme value process and its underlying response process. Then the joint probability density of the AMV can be evaluated by solving the Chapman-Kolmogorov Equation, e.g., via the path integral solution (PIS). Further, the PDF of the time-variant extreme value process is obtained, which and can be used, say, to estimate the dynamic reliability of a stochastic system. Finally For the purpose of illustration and verification, several numerical examples are illustrated studied and compared with Monte Carlo solution. Problems to be further studied are also discussed.
\end{abstract}

* Corresponding author. E-mail: chenjb@tongii.edu.cn (J. B. Chen).

E-mail address: lyumz@ tongii.edu.cn (M. Z. Lyu), antonina.pirrotta@unipa.it, Antonina.Pirrotta@liverpool.ac.uk (A. Pirrotta). 
Keywords: Time-variant extreme value process; Augmented Markov vector process; Đynamie reliability; Path integral solution (PIS); Stochastic dynamic system; Poisson white noise excitation.

\section{Introduction}

Poisson white noise excitations are not uncommon in science and engineering fields. In many practical problems, the dynamical systems of concern are usually enforced by pulse excitations which occurs sparsely with occurrence time and intensity being random. Poisson white noise processes are reasonable models in these cases, e.g., highway bridges under traffic loads [1,2], vehicles or truacks traveling on rough roads [3], buffeting airplanes tails [4], and earthquake excited structures [5], etc.

The probability density function (PDF) of the response for of stochastic dynamical systems enforced by Poisson white noise processes satisfies a class of generalized Kolmogorov equations, i.e., the Kolmogorov-Feller equation. It is difficult to capture the analytical-closed-form solution of its PDF, but the numerical results can be obtained by solving the Kolmogorov-Feller equation or the corresponding integral equation, Chapman-Kolmogorov equation using the numerical methods, e.g., the finite element method $[6,7]$, the spectral finite difference method [7 8$]$, the stochastic averaging method [89], the complex fractional moments method [ $9 \underline{10}]$, the generalized cell mapping method [11 $\theta$ ], and the path integral method [121-1 $\underline{5} 3]$, etc. Further, if the first-passage reliability for $\underline{\text { in terms }}$ of a certain specified threshold is of interest, an absorbing boundary condition can be applied imposed on the differential or integral equation that the PDF satisfies, namely, the probability density evolution equation, to yield the remaining probability density [1ㅁ-1 $\underline{6} 6]$. However, if the threshold is time variant, say due to the deterioration of materials in the long service life [197], or the reliability for-in terms of different thresholds is of concern, such treatment is not convenient. The remaining probability density needs to be calculated again and againrepeatedly under eachcorresponding absorbing boundary condition for different thresholds, which consumes prohibitively large computational efforts-will take a huge calculation cost.

An alternative approach for the first-passage reliability is to capture the probability distribution of the extreme value (e.g., maximum value, minimum value, and-or absolute maximum value) of the response in a certain-specified time period. For a long time gGreat challenges exist in, and intensive efforts have been devoted to the extreme value distributions (EVD) for long time 
$[18 \underline{20}, 19 \underline{21}]$. The limit or asymptotic distributions of random sampling/sequences in different cases were studied and available in literature [220]. However, even-much less understanding has been achieved for the EVD of stochastic processes in continuous time domain. Only some very special results were achieved for some particular stochastic processes [21-23-25]. Alternatively, some approximate approaches were studied based on the level-crossing process theory. However, in the level-crossing process theory, needed in the Rice formula is the joint probability density of the stochastic process and its derivative [264], which is usually unavailable for practical non-linear stochastic dynamical systems $[2 \underline{7} 5,2 \underline{8} 6]$. Besides, the assumptions on the property of crossingevents usually leads to extra errors. To circumvent the difficulties, a new approach was developed based on the probability density evolution method (PDEM) [286,297], by which the EVD can be evaluated through constructing a virtual stochastic process and calculating the PDF of its equivalent extreme-value event by PDEM [2830]. The structural system dynamic reliability can then be captured [2931]. However, the equivalent extreme-value event is constructed for a certain specified time interval. If the time-variant reliability is of interest, the equivalent extreme-value events for different time intervals should be constructed and thus huge computational costs are needed.

Besides the above approaches, for the purpose of capturing EVD, an extreme value process of a stochastic process, i.e., the value of this new process is the extreme value of the underlying stochastic process during the time interval from the initial time to the present time of concern, can be defined. Clearly, an extreme value process is, in the sense of sample, non-decreasing monotonous. As a result, the EVD of an extreme value process is ef coursecertainly also time variant. Once this

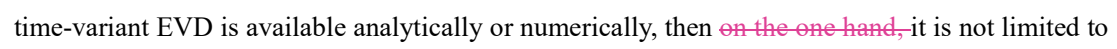
a specified time interval, and on the other handmoreover, it is not limited to the specified constant threshold. However, capturing the analytical solution of PDF of the time-variant extreme value process is still -difficult. Monte Carlo simulation (MCS), usually regarded as the final choice, is useful to obtain the EVD for systems of relatively low dimensions by numerically solving the Itô stochastic differential equation for each sample [320-3 $\underline{2} 3]$. But the computational cost for MCS is necessarily usually very high to ensure required accuracy. The augmented Markov vector process incorporated with the path integration solution (PIS) provides an alternative approach for solving the PDF of the time-variant extreme value process, and shows the effectiveness for low-dimensional stochastic dynamical systems enforced by Gaussian white noise [3 64 ]. 
Based on the above research, in this paper, the time-variant extreme value process of response of stochastic dynamical systems respense enforced by Poisson white noise is investigated. Although the time-variant extreme value process is not Markovian, an augmented Markov vector (AMV) process is constructed by combining the time-variant extreme value process and the underlying Markov response process. The numerical solution method via PIS based on the ChapmanKolmogorov equation are is then elaborated. Several examples, including linear and non-linear, onedimensional and multi-dimensional systems, are illustrated, showing the effectiveness of the proposed method. Problems to be further studied are also discussed.

\section{Poisson white noise excitation}

A Poisson white noise, $\xi_{\mathrm{P}}(t)$, can be defined as [375]

$$
\xi_{\mathrm{P}}(t)=\sum_{n=1}^{N(t)} \Gamma_{n} \delta\left(t-\widetilde{T}_{n}\right)
$$

in which $N(t)$ is a Poisson point process with the rate $\lambda$, i.e. $N(t) \sim \mathrm{P}(\lambda t) ; \delta(\cdot)$ is Dirac's delta function; $\widetilde{T}_{n}, n=1, \cdots, N(t)$ is the $n$-th realization of the Poisson random events; $\widetilde{T}_{n}=\sum_{i=1}^{n} T_{i}$, where $T_{i}, i=1, \cdots, N(t)$ are the time intervals between two adjacent Poisson random events, and they are independent random variables following exponential distribution with the mean value $\frac{1}{\lambda}$, i.e. $T_{i} \sim \operatorname{Exp}\left(\frac{1}{\lambda}\right) ; \Gamma_{n}, n=1, \cdots, N(t)$ are another set of random variables which have independent and identically distributions, and they are also independent to $T_{i}$ and ean may have-follow arbitrary known distribution whose PDF is denoted as $p_{\Gamma}(\gamma)$.

For clarity, firstly Cconsider a one-dimensional process $X(t)$ enforced by Poisson white noise. The equation of motion can be written as

$$
\dot{X}(t)=f[X(t), t]+\xi_{\mathrm{P}}(t)
$$

where $f(\cdot)$ is a deterministic function. Eq.(2)(2) can be rewritten into a stochastic differential equation 


$$
\mathrm{d} X(t)=f[X(t), t] \mathrm{d} t+\mathrm{d} W_{\mathrm{P}}(t)
$$

in which $W_{\mathrm{P}}(t)$ is a compound Poisson process, i.e.

$$
W_{\mathrm{P}}(t)=\int_{0}^{t} \xi_{\mathrm{P}}(\tau) \mathrm{d} \tau=\sum_{n=1}^{N(t)} \Gamma_{n} U\left(t-\widetilde{T}_{n}\right)=\sum_{n=1}^{N(t)} \Gamma_{n}
$$

where $U(\cdot)$ is the Heaviside's's unit step function, i.e., $U(x)=0$, if $x<0$ and $U(x)=1$, if $x \geqslant 0$.

Denote $\Delta W_{\mathrm{P}}(t)=W_{\mathrm{P}}(t+\Delta t)-W_{\mathrm{P}}(t)$, where $\Delta t$ is an arbitrary small time increment 带格式的：正文，缩进：首行缩进： 2 字符 at time instant $t$. According to the Poisson assumption, there is $\Delta W_{\mathrm{P}}(t)=W_{\mathrm{P}}(\Delta t)=0$ or $\Gamma$

$$
\Delta W_{\mathrm{P}}(t)=0 \text { or } \Gamma
$$

Wwhich means that if the Poisson event occur in the time interval $\Delta t$, the increment $\Delta W_{\mathrm{P}}(t)$ is a random variable with the distribution $p_{\Gamma}(\gamma)$; otherwise, the value of $\Delta W_{\mathrm{P}}(t)$ is zero. andTherefore, there is $\operatorname{Pr}\left\{\Delta W_{\mathrm{P}}(t)=0\right\}=1-\lambda \Delta t$, where $\operatorname{Pr}\{\cdot\}$ denotes the probability of event. In Eq.(5)(5), $\Gamma$ is a random variable whese with $\mathrm{PDF}$ is $p_{\Gamma}(\gamma)$. Therefore, if $W_{\mathrm{P}}(t)=w$, the probability that the value of $W_{\mathrm{P}}(t+\Delta t)$ will still be $w$ is $1-\lambda \Delta t$, and the probability that it will change to be $w+\Gamma$ is $\lambda \Delta t$. Hence, the process $W_{\mathrm{P}}(t)$ is Markovian, and its transition probability density (TPD) for small values of time increment $\tau$ can be written as

$$
p_{W_{\mathrm{r}}}\left(w, t+\tau \mid w^{\prime}, t\right)=(1-\lambda \tau) \delta\left(w-w^{\prime}\right)+\lambda \tau p_{\Gamma}\left(w-w^{\prime}\right)
$$

Further, it is easy to verify that the process $X(t)$ driven by Eq.(3)(3) is Markovian, and its PDF satisfies the Chapman-Kolmogorov equation, namely

$$
p_{X}(x, t+\tau)=\int_{-\infty}^{\infty} p_{X}\left(x^{\prime}, t\right) p_{X}\left(x, t+\tau \mid x^{\prime}, t\right) \mathrm{d} x^{\prime}
$$

in which $\tau$ is an arbitrary time increment; $p_{X}\left(x, t+\tau \mid x^{\prime}, t\right)$ is the transition probability density conditional on $X(t)=x^{\prime}$. According to Eq.(3) (3), for an arbitrary small time increment $\Delta t$, we have 


$$
X(t+\tau)=X(t)+f[X(t), t] \tau+\Delta W_{\mathrm{P}}(t)
$$

in which $\Delta W_{\mathrm{P}}(t)=W_{\mathrm{P}}(t+\tau)-W_{\mathrm{P}}(t)$-According to the definition of compound Poisson process, the probability of $\Delta W_{\mathrm{P}}(t)=0$, which means $X(t+\tau)=x^{\prime}+f\left(x^{\prime}, t\right) \tau$ is deterministic, is $1-\lambda \tau$; the probability of $\Delta W_{\mathrm{P}}(t)=\Gamma \quad$ is $\quad \lambda \tau \quad$ when $X(t+\tau)=x^{\prime}+f\left(x^{\prime}, t\right) \tau+\Gamma$ is a random variable. Therefore, the TPD of $X(t)$ can be written as $[13 \underline{z}]$

$$
p_{X}\left(x, t+\tau \mid x^{\prime}, t\right)=(1-\lambda \tau) \delta\left[x-x^{\prime}-f\left(x^{\prime}, t\right) \tau\right]+\lambda \tau p_{\Gamma}\left[x-x^{\prime}-f\left(x^{\prime}, t\right) \tau\right]
$$

Once the TPD of $X(t)$ is known, the PDF of $X(t)$ can be calculated according to Eq.(7)(7) step by step, which is the so-called path integral method [121].

\section{The time-variant extreme value process and augmented Markov vector} process

Consider a one-dimensional stochastic process $X(t)$. Define the time-variant extreme value process as

$$
Z(t)=\operatorname{ext}\{X(\tau), 0 \leqslant \tau \leqslant t\}
$$

in which $\operatorname{ext}\{\cdot\}$ denotes the extreme value of the bracketed quantity, which can be the maximum, the minimum, or the maximum absolute value, etc., depending on the practical issues of concern. It is easy to know that $Z(t)$ is also a stochastic process which is monotonous in the sense of sample. For instance, if $Z(t)$ is the maximum or maximum absolute value process, one arbitrary sample of $Z(t)$ is monotoneically undiminishednon-decreasing; if $Z(t)$ is the minimum value process, one arbitrary sample of $Z(t)$ is monotoneically non-increasing-incremental.

Such a time-dependent extreme value is of great significance in sciences and engineering fields. For instance, the excursion (first-passage) problem is widely encountered. In this case, the probability without any excursion (reliability) of the system is

$$
R(t)=\operatorname{Pr}\{X(\tau)<b, 0 \leqslant \tau \leqslant t\}
$$

\begin{tabular}{l|} 
域代码已更改 \\
域代码已更改 \\
域代码已更改 \\
域代码已更改
\end{tabular}


If $Z(t)$ is defined as the maximum value process of $X(t)$, namely

$$
Z(t)=\max \{X(\tau), 0 \leqslant \tau \leqslant t\}
$$

then the reliability function is given by

$$
R(t)=\int_{-\infty}^{b} p_{Z}(z, t) \mathrm{d} z
$$

in which $p_{Z}(z, t)$ is the PDF of process $Z(t)$. A differentiation of the failure probability function, which is the complementary of reliability function, in terms of time will yield further the PDF of the first excursion time [3 $\underline{8} 6]$.

Unfortmately However, as discussed in the preceding section, for a general process, it is difficult to obtain the analytical closed-form solution of the PDF of its time-variant extreme value process, except several very special conditions [164,_37-39-41]. HoweverFortunately, its PDF can be evaluated numerically, which is the main task of the present paper.

If $X(t)$ is a Markov process, the probabilistic information of $Z(t+\Delta t)$ for an infinitesimal time increment $\Delta t$ depends only on the information of $Z(t)$ and $X(t)$. For instance, for the maximum value process $Z(t)$, there is

$$
Z(t+\Delta t)= \begin{cases}Z(t), & Z(t)>X(t+\Delta t) \\ X(t+\Delta t), & Z(t) \leqslant X(t+\Delta t)\end{cases}
$$

Therefore, the time-variant extreme value process $Z(t)$ itself is not Markovian.

However, an augmented vector process $(Z(t), X(t))^{\mathrm{T}}$ can be constructed. It is known from Eq.(14)(14) that the probabilistic information of $(Z(t+\Delta t), X(t+\Delta t))^{\mathrm{T}}$ only depends only on the augmented vector process $(Z(t), X(t))^{\mathrm{T}}$ is Markovian. Though the analytical solution for the augmented Markov vector (AMV) process is still unavailable for general problems, numerical methods are feasible and will be elaborated in the following sections.

\section{The path integral solution of probability density function of the augmented}

\section{Markov vector process}




\subsection{The extreme value process of a scalar process}

According to the Markov property of the augmented vector process $(Z(t), X(t))^{\mathrm{T}}$, its joint PDF can be captured numerically using PIS by the Chapman-Kolmogorov equation, i.e.

$$
p_{Z X}(z, x, t+\tau)=\int_{-\infty}^{\infty} \int_{-\infty}^{\infty} p_{Z X}\left(z^{\prime}, x^{\prime}, t\right) p_{Z X}\left(z, x, t+\tau \mid z^{\prime}, x^{\prime}, t\right) \mathrm{d} z^{\prime} \mathrm{d} x^{\prime}
$$

in which $p_{Z X}(z, x, t)$ is the joint PDF of $(Z(t), X(t))^{\mathrm{T}} ; \tau>0$ is an arbitrary small time increment. According to Eq.(10)(10), the initial condition can be

$$
p_{Z X}(z, x, 0)=\delta(z-x) p_{X}(x, 0)
$$

where $p_{X}(x, 0)$ is the PDF of $X(0)$. If the initial value of the process $X(t)$ is deterministic, e.g., $X(0)=x_{0}$, then $p_{X}(x, 0)=\delta\left(x-x_{0}\right)$. Therefore, once the TPD $p_{Z X}\left(z, x, t+\tau \mid z^{\prime}, x^{\prime}, t\right)$ is known, $p_{Z X}(z, x, t)$ can be solved step by step numerically by Eq.(15)(15).

When the joint PDF $p_{Z X}(z, x, t)$ is obtained, the PDF of the time-variant extreme value process $Z(t)$ can be further obtained by

$$
p_{Z}(z, t)=\int_{-\infty}^{\infty} p_{Z X}(z, x, t) \mathrm{d} x
$$

Simultaneously, the PDF of $X(t)$ can also be obtained as a marginal PDF

$$
p_{X}(x, t)=\int_{-\infty}^{\infty} p_{Z X}(z, x, t) \mathrm{d} z
$$

According to the theorem of total probability, the TPD $p_{Z X}\left(z, x, t+\tau \mid z^{\prime}, x^{\prime}, t\right)$ can be given by

$$
p_{Z X}\left(z, x, t+\tau \mid z^{\prime}, x^{\prime}, t\right)=p_{Z \mid X}\left(z, t+\tau ; x \mid z^{\prime}, t\right) p_{X}\left(x, t+\tau \mid x^{\prime}, t\right)
$$

where $p_{Z \mid X}\left(z, t+\tau ; x \mid z^{\prime}, t\right)$ is denotese as the probability density of $Z(t)=z$ conditional on $\left\{Z(t)=z^{\prime}, X(t+\tau)=x\right\}$. Specifically, if $Z(t)$ is the maximum value process of process $X(t)$ as defined in Eq.(12) $(12)$, then according to Eq.(14) $(14)$, there are two different cases to be

设置了格式：字体颜色：蓝色

设置了格式：字体颜色：蓝色

设置了格式：字体颜色：蓝色 设置了格式: 字体颜色: 蓝色 
considered: (1) if $z^{\prime}>x$, there is $z=z^{\prime}$; (2) if $z^{\prime} \leq x$, there is $z=x$. Therefore, the conditional TPD $p_{Z \mid X}\left(z, t+\tau ; x \mid z^{\prime}, t\right)$ can be given by

$$
p_{Z \mid X}\left(z, t+\tau ; x \mid z^{\prime}, t\right)= \begin{cases}\delta\left(z-z^{\prime}\right), & z^{\prime}>x \\ \delta(z-x), & z^{\prime} \leqslant x\end{cases}
$$

Substituting Eq.(20)(20) in Eq.(19)(19) yields

$$
p_{Z X}\left(z, x, t+\tau \mid z^{\prime}, x^{\prime}, t\right)=\left[u\left(z^{\prime}-x\right) \delta\left(z-z^{\prime}\right)+u\left(x-z^{\prime}\right) \delta(z-x)\right] p_{X}\left(x, t+\tau \mid x^{\prime}, t\right)(21)
$$

设置了格式：字体颜色：蓝色

设置了格式：字体颜色：蓝色

where $u(\cdot)$ is Heaviside's unit step function; $p_{X}\left(x, t+\tau \mid x^{\prime}, t\right)$ is the TPD of $X(t)$. If $X(t)$ is a response process enforced by Poisson white noise, i.e., governed by Eq.(3)(3), then $p_{X}\left(x, t+\tau \mid x^{\prime}, t\right)$ can be obtained by Eq.(9)(9).

\subsection{The extreme value process of a vector process}

The similar ideas elaborated above can be extended to capture the PDF of the time-variant extreme value process of any arbitrary component of a multi-dimensional Markov vector process. Without loss of generality, consider an $n$-dimensional Markov vector process $\boldsymbol{X}(t)=\left(X_{1}(t), \cdots, X_{n}(t)\right)^{\mathrm{T}}$, where $X_{l}(t), l=1,2, \cdots, n$ are the components. Then the maximum value process of a component $X_{l}(t)$ can be defined as

$$
Z_{l}(t)=\max \left\{X_{l}(\tau), 0 \leqslant \tau \leqslant t\right\}
$$

Based on the similar reasoning in the preceding section, the augmented vector process $\left(Z_{l}(t), \boldsymbol{X}^{\mathrm{T}}(t)\right)^{\mathrm{T}}$ is an $(n+1)$-dimensional Markov vector process.

Denote the TPD of $\left(Z_{l}(t), \boldsymbol{X}^{\mathrm{T}}(t)\right)^{\mathrm{T}}$ as $p_{Z_{l} \boldsymbol{X}}\left(z_{l}, \boldsymbol{x}, t+\tau \mid z_{l}^{\prime}, \boldsymbol{x}^{\prime}, t\right)$, then Eq.(19)(19) and (20)(20) can be expended to multi-dimensional cases. Hence, there will be

$$
\begin{aligned}
& p_{Z_{l} \boldsymbol{X}}\left(z_{l}, \boldsymbol{x}, t+\tau \mid z_{l}^{\prime}, \boldsymbol{x}^{\prime}, t\right) \\
= & {\left[u\left(z_{l}^{\prime}-x_{l}\right) \delta\left(z_{l}-z_{l}^{\prime}\right)+u\left(x_{l}-z_{l}^{\prime}\right) \delta\left(z_{l}-x_{l}\right)\right] p_{\boldsymbol{X}}\left(\boldsymbol{x}, t+\tau \mid \boldsymbol{x}^{\prime}, t\right) }
\end{aligned}
$$

in which $p_{\boldsymbol{X}}\left(\boldsymbol{x}, t+\tau \mid \boldsymbol{x}^{\prime}, t\right)$ is the TPD of $\boldsymbol{X}(t)$. 
Therefore, once the TPD of $\left(Z_{l}(t), \boldsymbol{X}^{\mathrm{T}}(t)\right)^{\mathrm{T}}$ has been obtained, the joint PDF can be calculated numerically by PIS, namely

$$
p_{Z_{l} \boldsymbol{X}}\left(z_{l}, \boldsymbol{x}, t+\tau\right)=\int_{-\infty}^{\infty} \int_{\mathbb{R}^{n}} p_{Z_{l} \boldsymbol{X}}\left(z_{l}^{\prime}, \boldsymbol{x}^{\prime}, t\right) p_{Z_{l} \boldsymbol{X}}\left(z_{l}, \boldsymbol{x}, t+\tau \mid z_{l}^{\prime}, \boldsymbol{x}^{\prime}, t\right) \mathrm{d} z_{l}^{\prime} \mathrm{d} \boldsymbol{x}^{\prime}
$$

The initial condition is

$$
p_{Z_{l} \boldsymbol{X}}\left(z_{l}, \boldsymbol{x}, 0\right)=\delta\left(z_{l}-x_{l}\right) p_{\boldsymbol{X}}(\boldsymbol{x}, 0)
$$

where $p_{\boldsymbol{X}}(\boldsymbol{x}, 0)$ is the PDF of $\boldsymbol{X}(0)$. If the initial value of $\boldsymbol{X}(t)$ is deterministic, e.g. $\boldsymbol{X}(0)=\boldsymbol{x}_{0}$, then $p_{\boldsymbol{X}}(\boldsymbol{x}, 0)=\delta\left(\boldsymbol{x}-\boldsymbol{x}_{0}\right)$.

Furthermore, the PDF of $Z_{l}(t)$ can be obtained by

$$
p_{Z_{l}}\left(z_{l}, t\right)=\int_{\mathbb{R}^{n}} p_{Z_{l} \boldsymbol{X}}\left(z_{l}, \boldsymbol{x}, t\right) \mathrm{d} \boldsymbol{x}
$$

Then the time-variant reliability, if interested, can be evaluated by Eq.(13)(13).

设置了格式: 字体颜色: 蓝色

The specific procedure of numerical implementation for PIS of time-variant extreme value process was elaborated by Chen and Lyu [364] and thus will not be repeated to avoid lengthiness. The above method will be illustrated below through several numerical examples.

For convenience, the proposed method for obtaining the PDF of the maximum value process can be called the augmented Markov vector process method in conjunction with PIS (AMV-PIS for short).

\section{Numerical examples}

\subsection{Uniform compound Poisson process}

Firstly, the maximum value process of a compound Poisson process $W_{\mathrm{P}}(t)$ is considered. The sample process is governed by Eq.(4)(4) [375]. Let $\Gamma_{n}, n=1, \cdots, N(t)$ obey the uniform distribution, i.e., $\Gamma_{n} \sim \mathrm{U}(-a, a)$, in which $a$ is a constant. For an arbitrary small time increment, the TPD of $W_{\mathrm{P}}(t)$ can be written by

$$
p_{W_{\mathrm{P}}}\left(w, t+\tau \mid w^{\prime}, t\right)=(1-\lambda \tau) \delta\left(w-w^{\prime}\right)+\frac{\lambda \tau u\left(a-\left|w-w^{\prime}\right|\right)}{2 a}
$$


If $Z(t)$ is considered as the maximum value process of $W_{\mathrm{P}}(t)$, according to $\mathrm{Eq} \cdot(21)(21)$, the TPD of augment vector process $\left(Z(t), W_{\mathrm{P}}(t)\right)^{\mathrm{T}}$ is governed by

$$
\begin{aligned}
& p_{Z W_{\mathrm{p}}}\left(z, w, t+\tau \mid z^{\prime}, w^{\prime}, t\right) \\
= & {\left[u\left(z^{\prime}-w\right) \delta\left(z-z^{\prime}\right)+u\left(w-z^{\prime}\right) \delta(z-w)\right] } \\
& \times\left[(1-\lambda \tau) \delta\left(w-w^{\prime}\right)+\frac{\lambda \tau u\left(a-\left|w-w^{\prime}\right|\right)}{2 a}\right]
\end{aligned}
$$

Therefore, the joint PDF $p_{Z W_{\mathrm{r}}}(z, w, t)$ of $\left(Z(t), W_{\mathrm{P}}(t)\right)^{\mathrm{T}}$ can be captured using the proposed AMV-PIS by Eq.(15)(15) and (16)(16), then the PDF $p_{Z}(z, t)$ of $Z(t)$ can be obtained.

In this case, the rate of the Poisson process takes $\lambda=3$; the constant is $a=1$; the time step is $\Delta t=0.02$; the solving domains are $z \in[0,15]$ and $x \in[-15,15]$; the grid sizes are $\Delta z=\Delta x=0.03$. The PDF surfaces of $W_{\mathrm{P}}(t)$ and $Z(t)$ against time $t \in[4,10]$ obtained by the proposed numerical method elaborated in Sec.4 are shown in Fig.1.
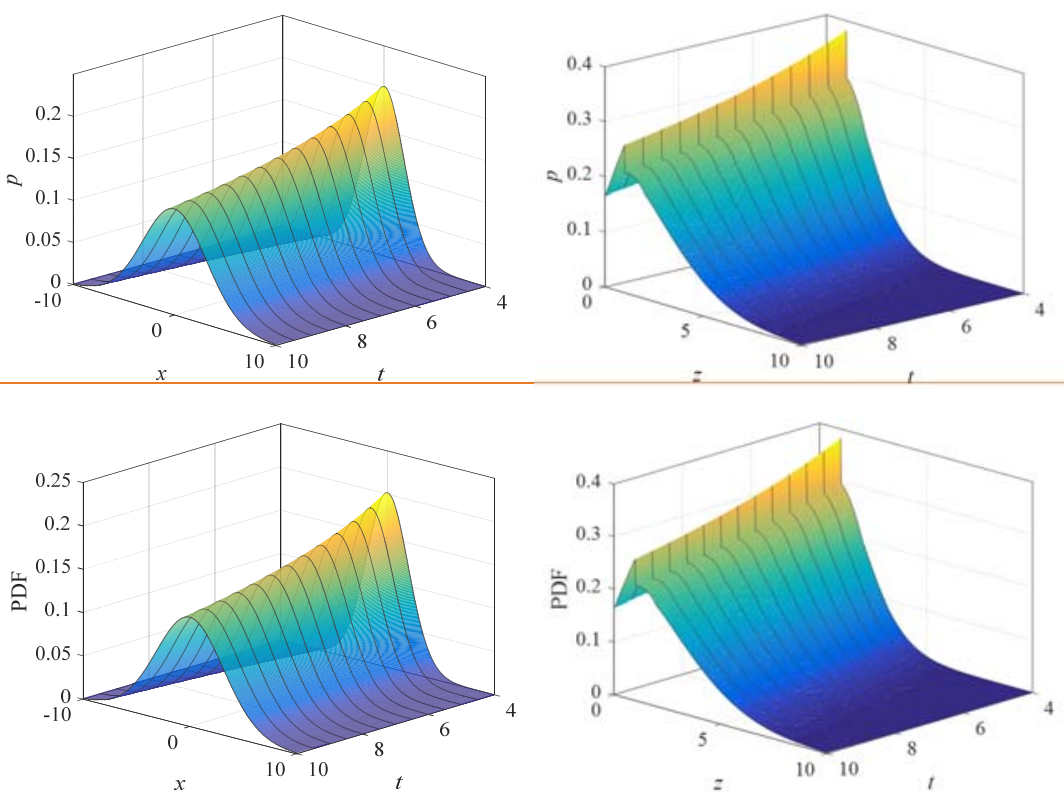

(a)

(b) 
Fig.1. The PDF surfaces of $W_{\mathrm{P}}(t)$ and $Z(t)$ of the uniform compound Poisson process. (a) PDF surface of $W_{\mathrm{P}}(t)$; (b) PDF surface of $Z(t)$.

At the time instant $t=10$, the comparison between the numerical results using the proposed AMV-PIS and Monte-Carlo simulation (MCS) in terms of the PDF and the cumulative distribution function (CDF) of $W_{\mathrm{P}}(t)$ is shown in Fig.2, in which $10^{5} 10^{6}$ samples are performed in the MCS to generate the histogram
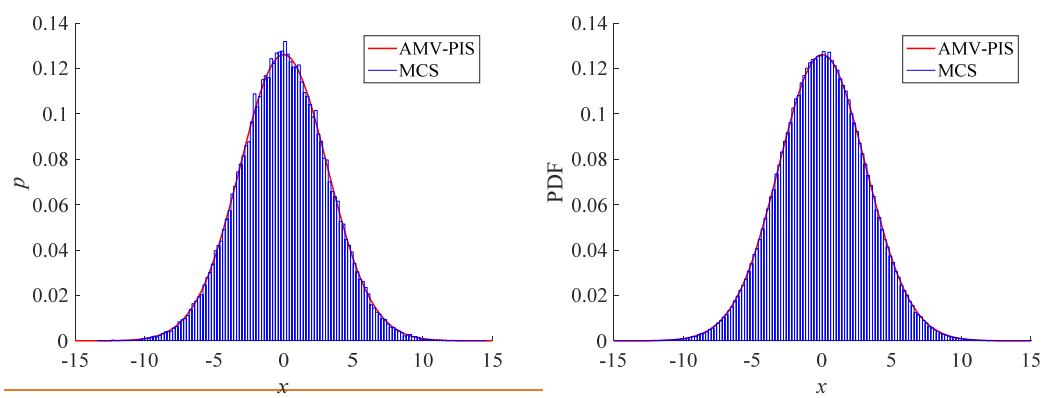

(a)
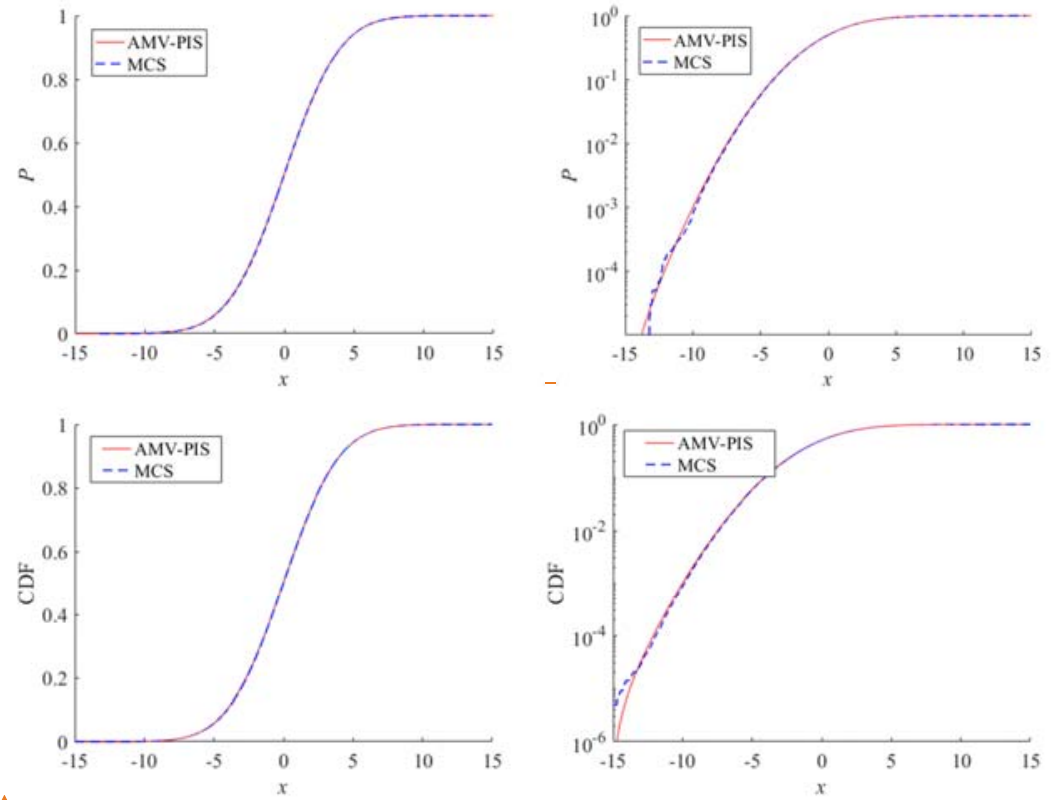

设置了格式：字体：加粗 
Fig.2. The comparison between AMV-PIS and MCS in terms of the PDF and CDF of the uniform compound Poisson process $W_{\mathrm{P}}(t)$ at $t=10$. (a) PDF; (b) CDF (in linear coordinates); (c) CDF (in logarithmic coordinates).

Simultaneously, the comparison between the numerical results using the proposed AMS-PIS MCS of the PDF and CDF of $Z(t)$ is shown in Fig.3, in which $10^{5} 10^{6}$ samples are performed in the MCS as well to generate the histogram. Perfect agreement is observed.
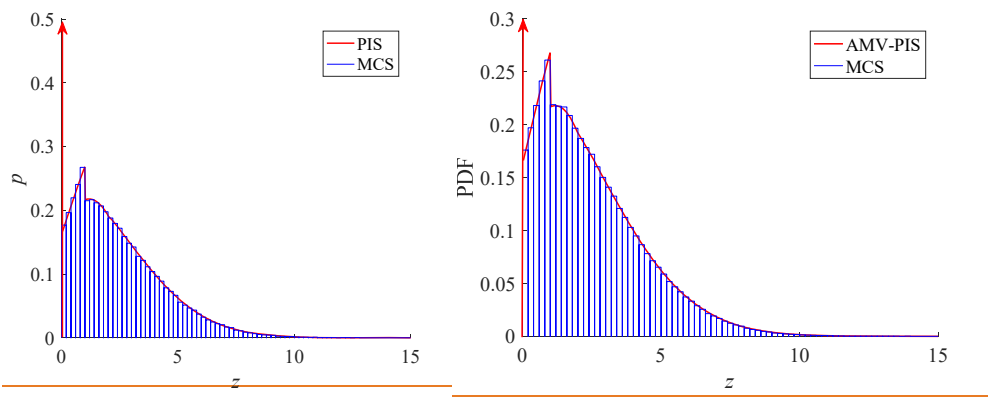

(a)
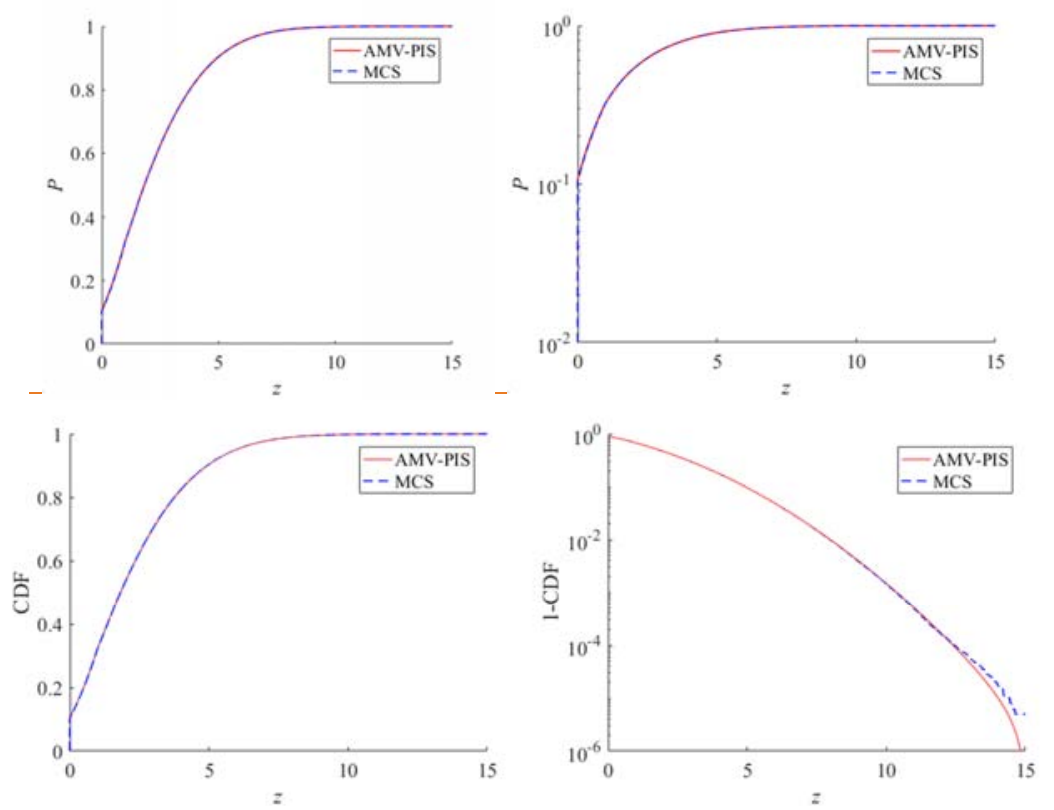
Fig.3. The comparison between AMV-PIS and MCS of $i$ terms of the PDF and CDF of the maximum value process $Z(t)$ of the uniform compound Poisson process at $t=10$. (a) PDF; (b) CDF (in linear coordinates); (c) 1 -CDF (in logarithmic coordinates)

It's noted that at the point $z=0$ there is a jump in the CDF of $Z(t)$, which means the PDF is a Dirac's delta value at the point $z=0$. This is also shown in Fig. 3 as a spike (arrow) at the origin with the value identical to the jump of CDF at the origin. Noting that $10^{6}$ times of MCS will yield accuracy in the tails in the order of magnitude of $10^{-4}$ in a confidence of $10 \%$ coefficient of variation. From Eq.3 it is seen that the accuracy of the proposed method reaches the order of magnitude of $10^{-4}$.

The PDFs of $Z(t)$ at some typical time instants are shown in Fig.4.
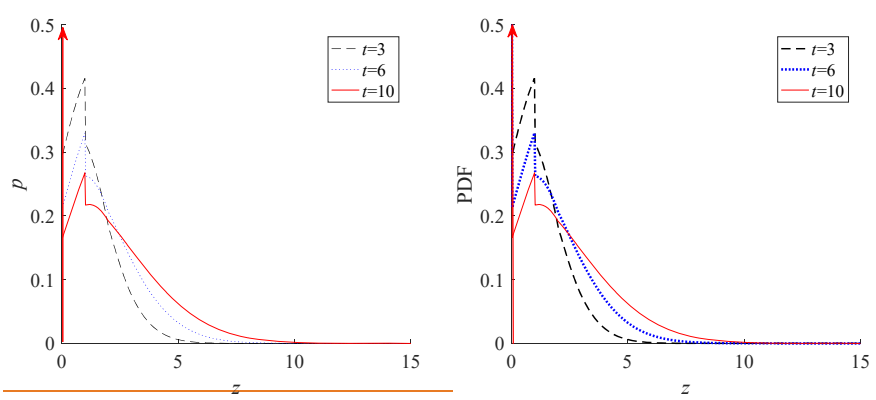

Fig.4. The PDFs of $Z(t)$ of the uniform compound Poisson process at different time instants.

It is observed that the PDFs of maximum value process becomes wider and lower as the time increase, which is consistent with the diffusion property of compound Poisson process. Remarkably, there is a jump in the PDF curve at $z=1$. This is because $\Gamma_{n}, n=1, \cdots, N(t)$ vary in the range of $[-1,1]$. Besides, $Z \geqslant 0$ holds with probability one at any time, though the value of $X$ can be positive or negative. Such property verifies the results also qualitatively. 


\subsection{Gaussian compound Poisson process}

Consider $W_{\mathrm{P}}(t)$ as a compound Poisson process whose sample process is governed by Eq.(4)(4) as well, but $\Gamma_{n}, n=1, \cdots, N(t)$ follow Gaussian distribution, i.e., $\Gamma_{n} \sim \mathrm{N}\left(0, \sigma^{2}\right)$, in which $\sigma$ is the standard deviation. In this case, for an arbitrary small time increment, the TPD of $W_{\mathrm{P}}(t)$ can be written by

$$
p_{W_{\mathrm{P}}}\left(w, t+\tau \mid w^{\prime}, t\right)=(1-\lambda \tau) \delta\left(w-w^{\prime}\right)+\frac{\lambda \tau}{\sqrt{2 \pi} \sigma} e^{-\frac{\left(w-w^{\prime}\right)^{2}}{2 \sigma^{2}}}
$$

If $Z(t)$ is considered as the maximum value process of $W_{\mathrm{P}}(t)$, according to Eq. $(21)(21)$, the TPD of augment vector process $\left(Z(t), W_{\mathrm{P}}(t)\right)^{\mathrm{T}}$ is governed by

$$
\begin{aligned}
& p_{Z W_{\mathrm{P}}}\left(z, w, t+\tau \mid z^{\prime}, w^{\prime}, t\right) \\
= & {\left[u\left(z^{\prime}-w\right) \delta\left(z-z^{\prime}\right)+u\left(w-z^{\prime}\right) \delta(z-w)\right] } \\
& \times\left[(1-\lambda \tau) \delta\left(w-w^{\prime}\right)+\frac{\lambda \tau}{\sqrt{2 \pi} \sigma} e^{-\frac{\left(w-w^{\prime}\right)^{2}}{2 \sigma^{2}}}\right]
\end{aligned}
$$

Therefore, the joint PDF $p_{Z W_{\mathrm{P}}}(z, w, t)$ of $\left(Z(t), W_{\mathrm{P}}(t)\right)^{\mathrm{T}}$ can be calculated using the proposed AMV-PIS by Eq.(15)(15) and (16)(16), then the PDF $p_{Z}(z, t)$ of $Z(t)$ can be obtained.

In the following numerical illustration, the rate is taken also as $\lambda=3$; the standard deviation is $\sigma=0.4$; the time step is $\Delta t=0.02$; the solving domains are $z \in[0,15]$ and $x \in[-15,15]$; the grid sizes are $\Delta z=\Delta x=0.03$. The PDF surfaces of $W_{\mathrm{P}}(t)$ and $Z(t)$ against time $t \in[4,10]$ obtained by the proposed numerical method are shown in Fig.5.
设置了格式：字体颜色：蓝色

设置了格式：字体颜色：蓝色

设置了格式：字体颜色：蓝色

设置了格式: 字体颜色：蓝色 

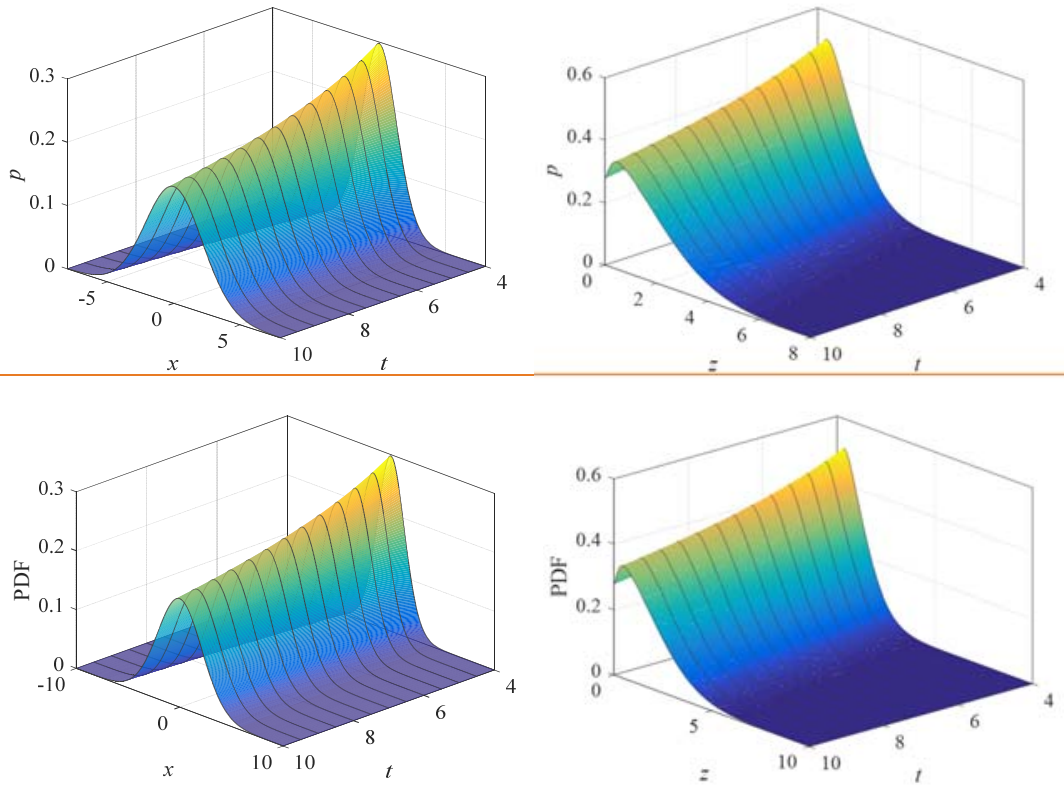

(a)

(b)

Fig.5. The PDF surfaces of $W_{\mathrm{P}}(t)$ and $Z(t)$ of the Gaussian compound Poisson process. (a) PDF surface of $W_{\mathrm{P}}(t) ;(\mathrm{b})$ PDF surface of $Z(t)$

At the time instant $t=10$, the comparison between the numerical results using the proposed method (AMV-PIS) and MCS in terms of the PDF and CDF of $W_{\mathrm{P}}(t)$ is shown in Fig.6, where $10^{5}-10^{6}$ _samples are performed in the MCS to generate the histogram.
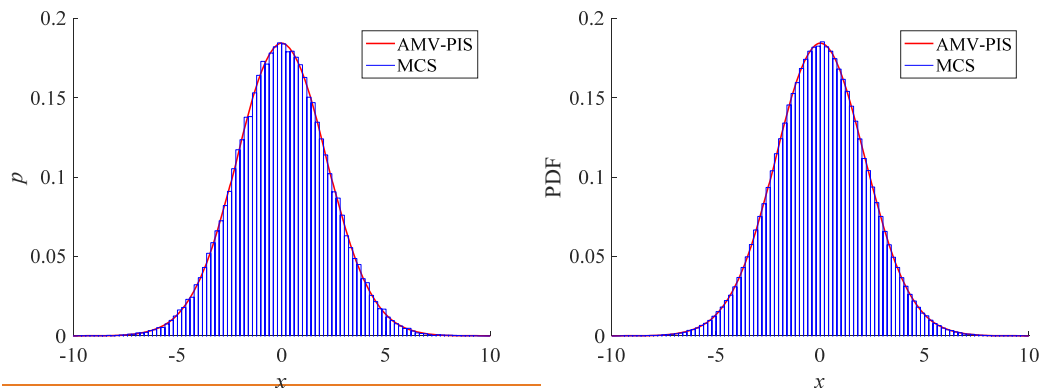

(a)

16
带格式的：两端对齐

\section{域代码已更改}



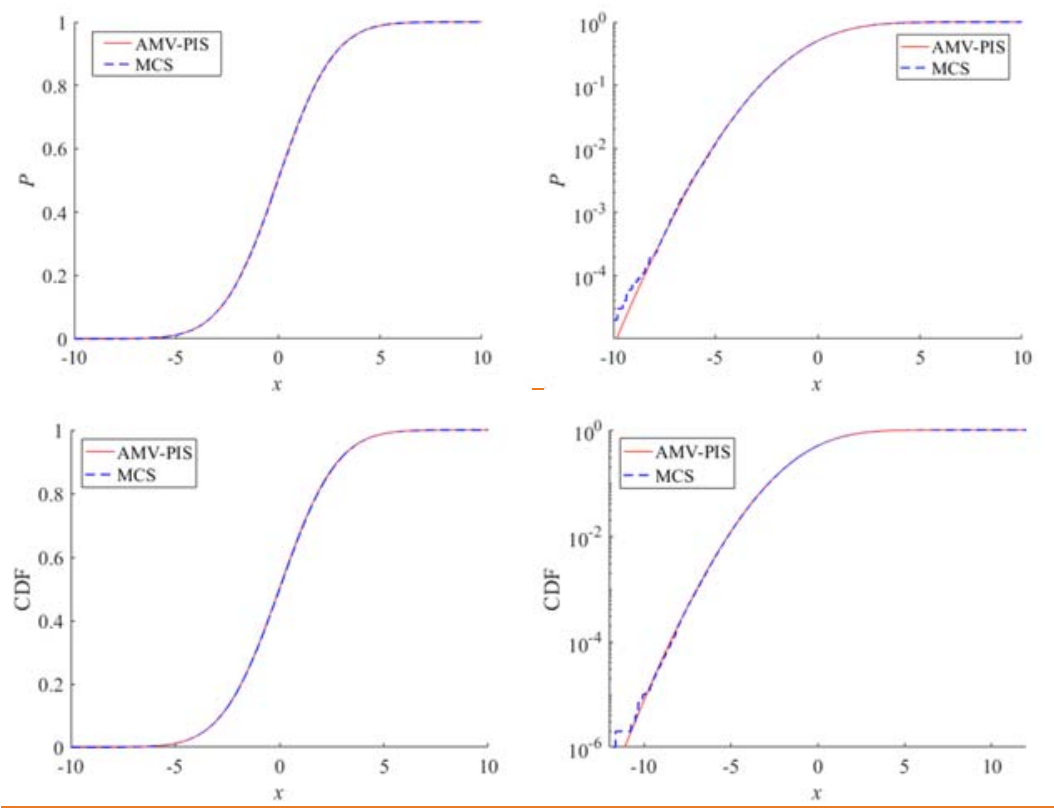

(b)

(c)

Fig.6. The comparison between AMV-PIS and MCS of the PDF and CDF in terms of the Gaussian compound Poisson process $W_{\mathrm{P}}(t)$ at $t=10$. (a) PDF; (b) CDF (in linear coordinates); (c) CDF (in logarithmic coordinates).

Simultaneously, the comparison between the numerical results using the proposed AMV-PIS

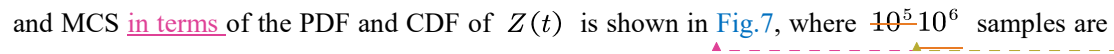
performed in the MCS as well. Again perfect agreement is observed in Figs.6 and 7. Besides, 设置了格式：字体：非加粗 域代码已更改 compared to Fig. 4(a), it is noted that there is no jump (discontinuity) at the PDF in the range $z>0$. However, similarly, at the point $z=0$ there is a concentrated probability in the PDF and thus a finite jump in the CDF. Interestingly, the finite jump of $\mathrm{CDF}$ at the origin is the same as in Fig. 3. This is because the occurrence rate of the two Poisson process are the same, though the distributions of the random variables are different, and the CDFs in Figs. 7 and 3 are at the same time instance of $t=10$. Again, this consistence between the two figures verifies the numerical results quantitatively. 

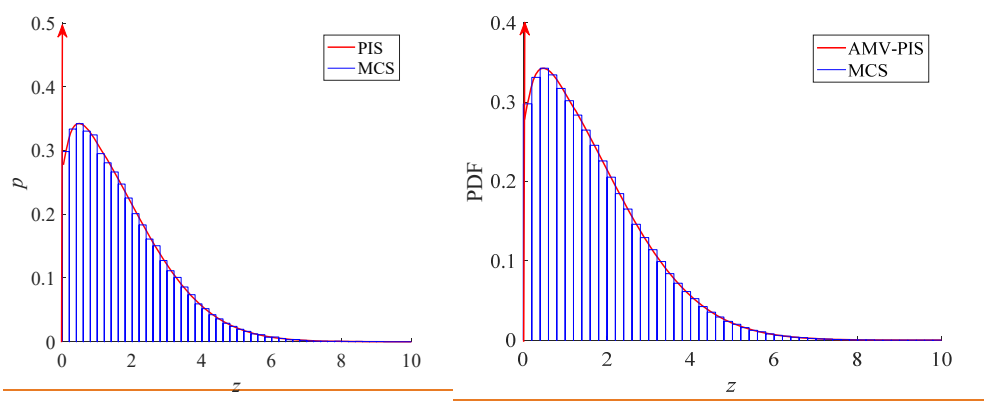

(a)
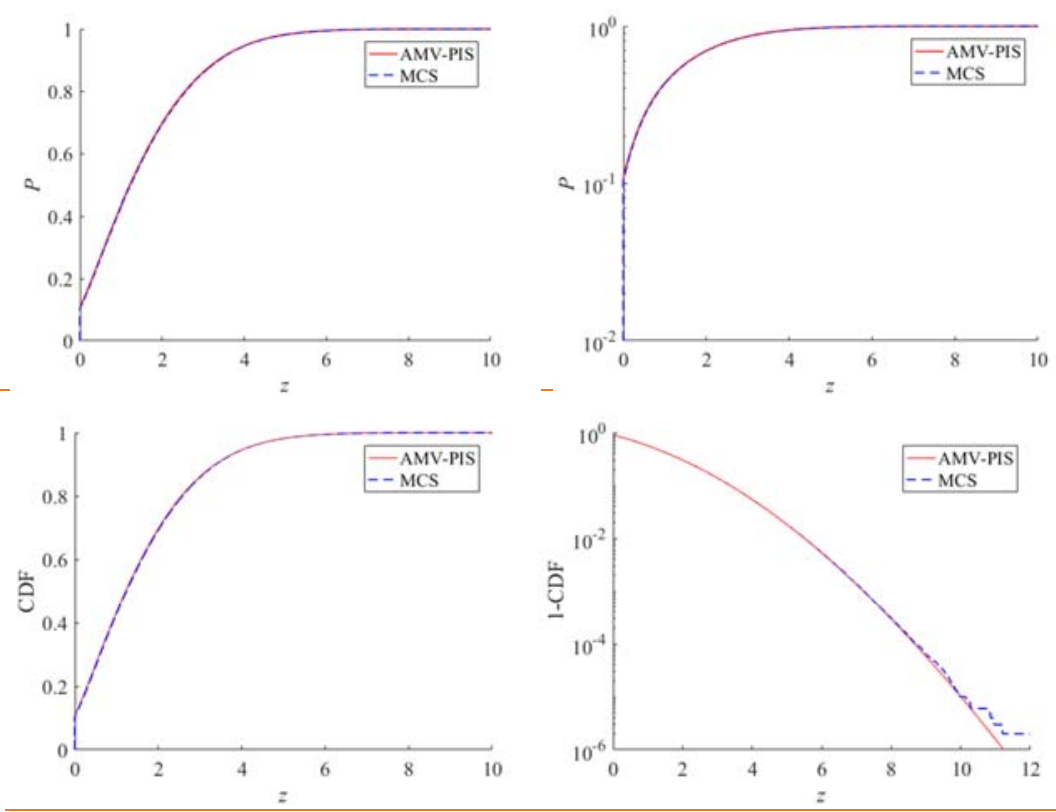

(b)

(c)

Fig.7. The comparison between AMV-PIS and MCS in terms of the PDF and CDF of the maximum value process

$Z(t)$ of the Gaussian compound Poisson process at $t=10$. (a) PDF; (b) CDF (in linear coordinates); (c) $\underline{1}$-CDF (in logarithmic coordinates).

Similarly, from Fig. 7 it is observed that the accuracy of the proposed method reaches the order 带格式的：缩进：首行缩进：2 字符 of magnitude of $10^{-4} \sim 10^{-5}$.

\subsection{One-dimensional non-linear process enforced by uniform Poisson white noise}


Consider a one-dimensional process $X(t)$ determined by the following stochastic differential equation $[1 \underline{3 z}]$

$$
\mathrm{d} X(t)=-\left[\alpha X(t)+\varepsilon X^{3}(t)\right] \mathrm{d} t+\mathrm{d} W_{\mathrm{P}}(t)
$$

where $\alpha$ is the linear coefficient; $\varepsilon>0$ is the non-linear coefficient; $W_{\mathrm{P}}(t)$ is the uniform Poisson white noise, whose sample process is governed by Eq.(4)(-4), where $\Gamma_{n}, n=1, \cdots, N(t)$ follow uniform distributions, i.e., $\Gamma_{n} \sim \mathrm{U}(-a, a)$, in which $a$ is a constant. For an arbitrary small time increment, according to Eq.(9)( $(9)$, the TPD of $X(t)$ can be written by

$$
\begin{aligned}
& p_{X}\left(x, t+\tau \mid x^{\prime}, t\right) \\
= & (1-\lambda \tau) \delta\left[x-(1-\alpha \tau) x^{\prime}+\varepsilon \tau x^{\prime 3}\right]+\frac{\lambda \tau u\left[a-\left|x-(1-\alpha \tau) x^{\prime}+\varepsilon \tau x^{\prime 3}\right|\right]}{2 a}
\end{aligned}
$$

If $Z(t)$ is considered as the maximum value process of $X(t)$ defined by Eq.(12)(12), then according to Eq. $(21)(21)$, the TPD of the augment vector process $(Z(t), X(t))^{\mathrm{T}}$ can be written by

$$
\begin{aligned}
& p_{Z X}\left(z, x, t+\tau \mid z^{\prime}, x^{\prime}, t\right) \\
= & {\left[u\left(z^{\prime}-x\right) \delta\left(z-z^{\prime}\right)+u\left(x-z^{\prime}\right) \delta(z-x)\right] } \\
& \times\left\{(1-\lambda \tau) \delta\left[x-(1-\alpha \tau) x^{\prime}+\varepsilon \tau x^{\prime 3}\right]+\frac{\lambda \tau u\left[a-\left|x-(1-\alpha \tau) x^{\prime}+\varepsilon \tau x^{\prime 3}\right|\right]}{2 a}\right\}
\end{aligned}
$$

Therefore, the joint PDF $p_{Z X}(z, x, t)$ of $(Z(t), X(t))^{\mathrm{T}}$ can be captured using the proposed AMV-PIS by Eq.(15)(15) and (16)(16), then the PDF $p_{Z}(z, t)$ of $Z(t)$ can be further obtained by a marginal integral.

In the present example, the initial value is taken as $x_{0}=0$; the occurrence rate is $\lambda=3$; the linear coefficient is $\alpha=2$; the non-linear coefficient is $\varepsilon=0.3$; the constant is $a=1$; the time step is $\Delta t=0.01$; the solving domains are $z \in[0,5]$ and $x \in[-5,5]$; the grid sizes are $\Delta z=\Delta x=0.01$. The PDF surfaces of $X(t)$ and $Z(t)$ against time $t \in[2,5]$ obtained by the proposed numerical method are shown in Fig.8.

设置了格式：字体颜色：蓝色

设置了格式：字体颜色：蓝色

设置了格式：字体颜色：蓝色

设置了格式：字体颜色：蓝色
设置了格式：字体颜色：蓝色 设置了格式：字体颜色：蓝色 

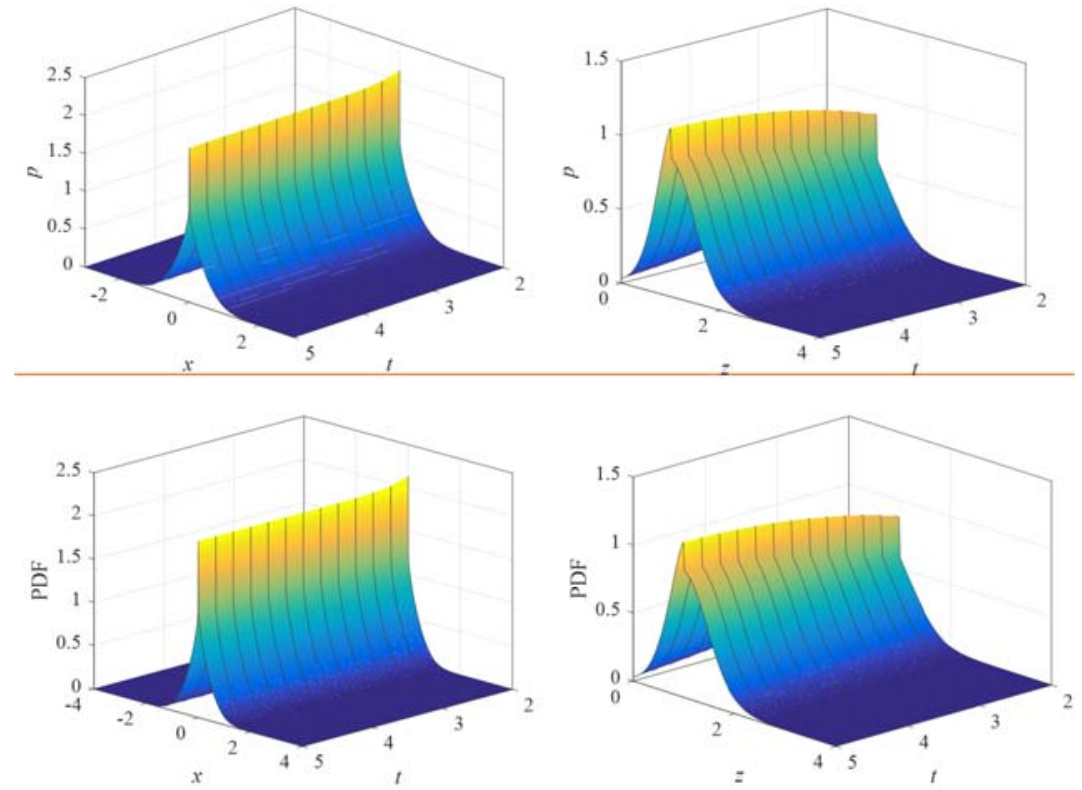

(a)

(b)

Fig.8. The PDF surfaces of $X(t)$ and $Z(t)$ of the one-dimensional non-linear process. (a) PDF surface of $X(t)$; (b) PDF surface of $Z(t)$.

At the time instant $t=5$, the comparison between the numerical results using the proposed AMV-PIS and MCS in terms of the PDF and CDF of $X(t)$ is shown in Fig.9, where $10^{5} 10^{6}$ samples are performed in the MCS. These results are also consistent with those obtained in Di Paola and Santoro [132]].
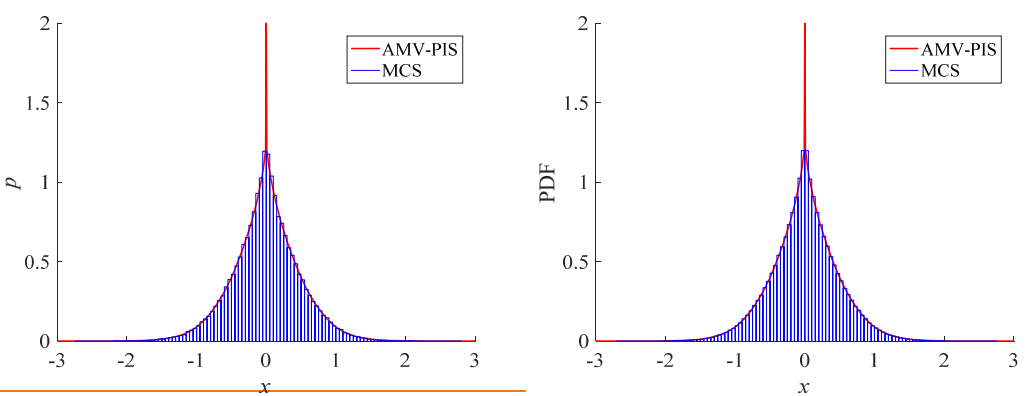

20
带格式的：两端对齐

域代码已更改 
(a)
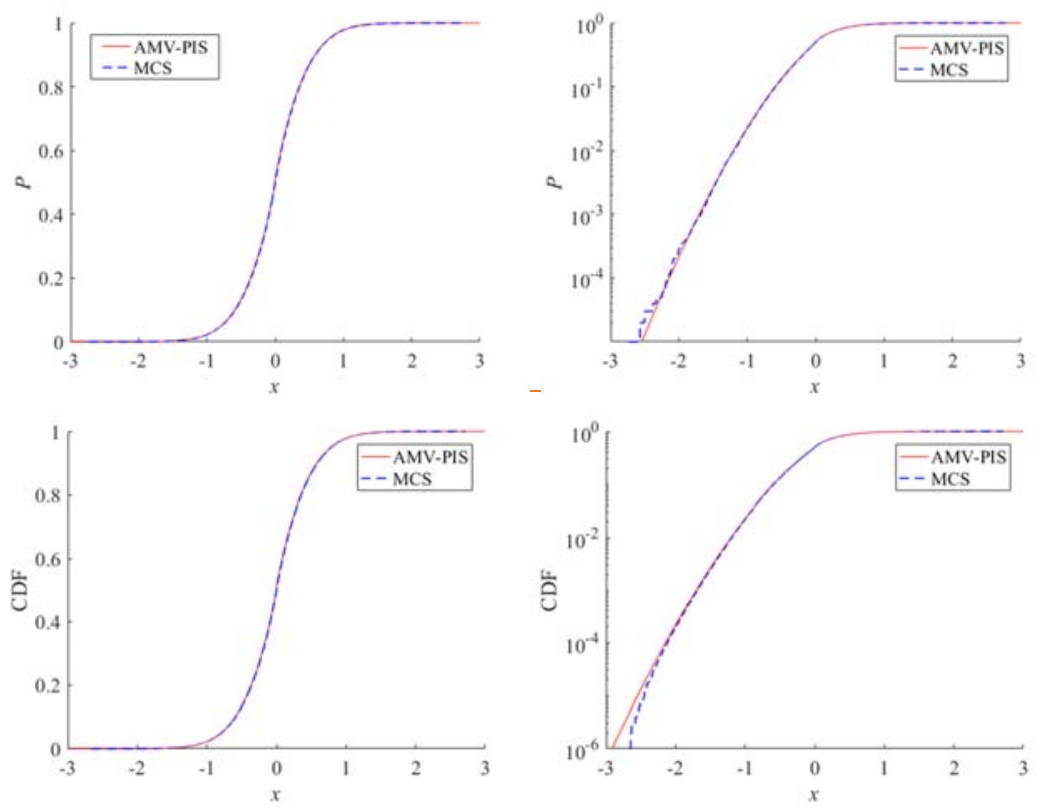

(b)

(c)

Fig.9. The comparison between AMV-PIS and MCS of the PDF and CDF of the one-dimensional non-linear process $X(t)$ at $t=5$. (a) PDF; (b) CDF (in linear coordinates); (c) CDF (in logarithmic coordinates).

Simultaneously, the comparison between the numerical results using AMV-PIS and MCS $\underline{\text { in }}$ terms of the PDF and CDF of $Z(t)$ is shown in Fig.10, where $10^{5} 10^{6}$ samples are performed in the MCS as well. Interestingly, there is also a discontinuity in the PDF in, and a finite jump is observed in the CDF of $Z$ at the time $t=0$ in Fig. 10(c). 

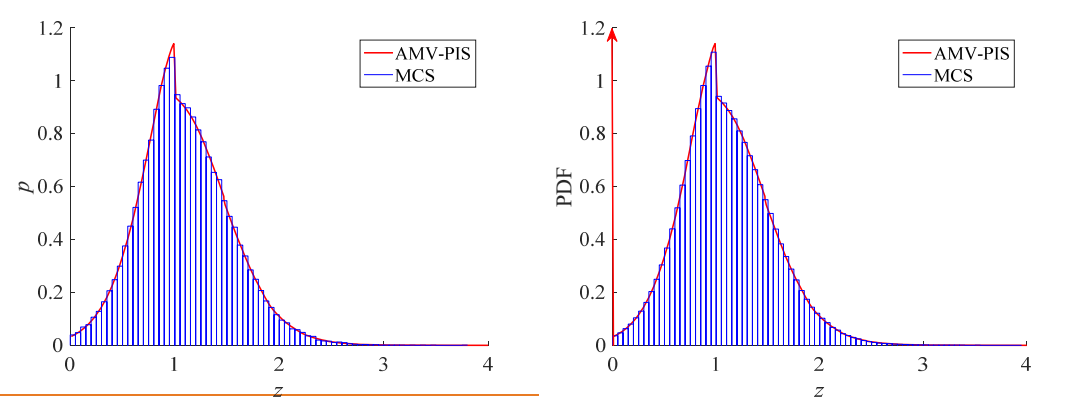

(a)
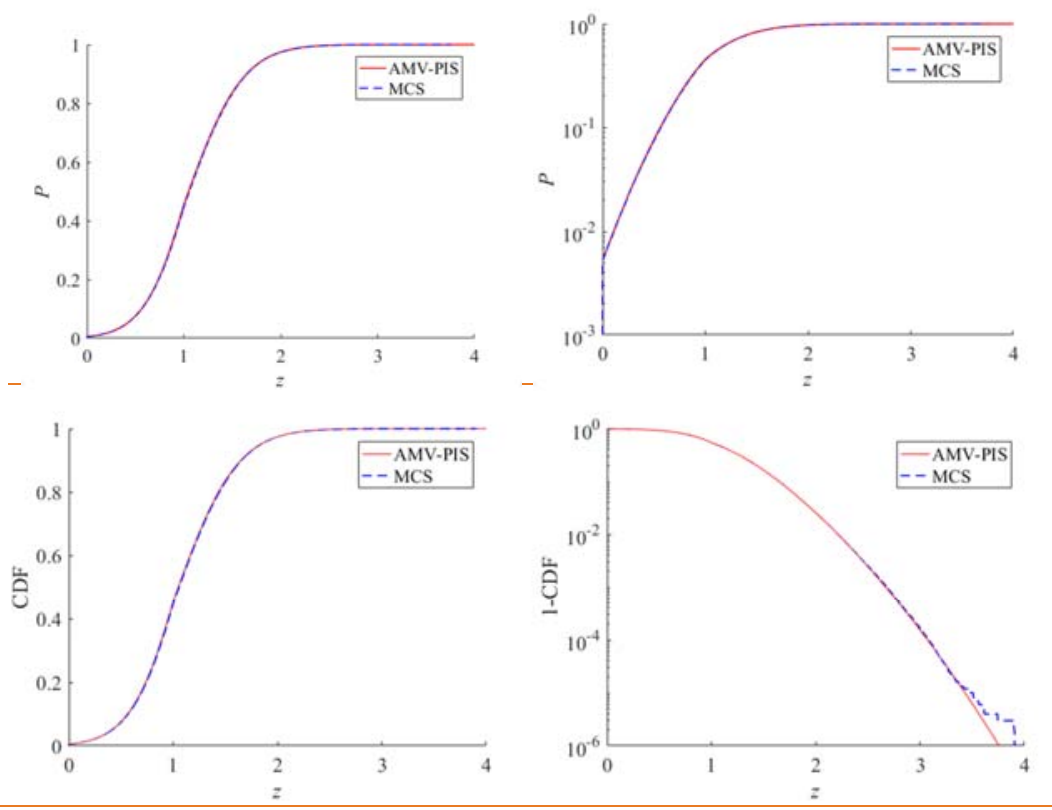

(b)

(c)

Fig.10. The comparison between AMV-PIS and MCS in terms of the PDF and CDF of the maximum value process $Z(t)$ of the one-dimensional non-linear process at $t=5$. (a) PDF; (b) CDF (in linear coordinates); (c) 1-CDF (in logarithmic coordinates).

\subsection{Duffing oscillator enforced by Gaussian Poisson white noise}

Finally, consider a non-linear, Gaussian Poisson white noise excited, two-dimensional systems, the Duffing oscillator [420]. The equation of motion is written as 


$$
\ddot{X}(t)+c \dot{X}(t)+\left[1+\varepsilon X^{2}(t)\right] X(t)=\xi_{\mathrm{P}}(t)
$$

in which $c$ is the linear damping coefficient; $\varepsilon>0$ is the non-linear stiffness coefficient; $\xi_{\mathrm{P}}(t)$ is the Gaussian Poisson white noise, whose sample process is governed by Eq.(1)(1), in which $\Gamma_{n}, n=1, \cdots, N(t)$ follow Gaussian distributions, i.e., $\Gamma_{n} \sim \mathrm{N}\left(0, \sigma^{2}\right)$, where $\sigma$ is the standard deviation. Denote $\dot{X}(t)=V(t)$, then the oscillator can be regarded as a two-dimensional Markov vector process $\boldsymbol{Y}(t)=(X(t), V(t))^{\mathrm{T}}$, whose stochastic differential equation can be written as

$$
\begin{gathered}
\mathrm{d} X(t)=V(t) \mathrm{d} t \\
\mathrm{~d} V(t)=-\left[X(t)+\gamma V(t)+\varepsilon X^{3}(t)\right] \mathrm{d} t+\mathrm{d} W_{\mathrm{P}}(t)
\end{gathered}
$$

in which $W_{\mathrm{P}}(t)$ is the corresponding Gaussian compound Poisson process. For an arbitrary small time increment $\tau$, the TPD of $\boldsymbol{Y}(t)$ can be written as [1 $\underline{1} z \underline{z}]$

$$
\begin{aligned}
& p_{\boldsymbol{Y}}\left(x, v, t+\tau \mid x^{\prime}, v^{\prime}, t\right) \\
= & \delta\left(x-x^{\prime}-\tau v^{\prime}\right) \\
& \times\left\{(1-\lambda \tau) \delta\left[v+\tau x^{\prime}-(1-\gamma \tau) v^{\prime}+\varepsilon \tau x^{\prime 3}\right]+\frac{\lambda \tau}{\sqrt{2 \pi} \sigma} e^{-\frac{\left[v+\tau x^{\prime}-(1-\gamma \tau) v^{\prime}+\varepsilon \tau x^{\prime 3}\right]^{2}}{2 \sigma^{2}}}\right\}
\end{aligned}
$$

If $Z(t)$ is considered as the maximum value process of $X(t)$, which is defined by Eq.(12)(12), according to Eq.(23)(23), the TPD of augment vector process $\left(Z(t), \boldsymbol{Y}^{\mathrm{T}}(t)\right)^{\mathrm{T}}$ is governed by

$$
\begin{aligned}
& p_{Z \mathbf{Y}}\left(z, x, v, t+\tau \mid z^{\prime}, x^{\prime}, v^{\prime}, t\right) \\
= & {\left[u\left(z^{\prime}-x\right) \delta\left(z-z^{\prime}\right)+u\left(x-z^{\prime}\right) \delta(z-x)\right] \delta\left(x-x^{\prime}-\tau v^{\prime}\right) } \\
& \times\left\{(1-\lambda \tau) \delta\left[v+\tau x^{\prime}-(1-\gamma \tau) v^{\prime}+\varepsilon \tau x^{\prime 3}\right]+\frac{\lambda \tau}{\sqrt{2 \pi} \sigma} e^{-\frac{\left[v+\tau x^{\prime}-(1-\tau) v^{\prime}+\varepsilon \tau x^{\prime 3}\right]^{2}}{2 \sigma^{2}}}\right\}
\end{aligned}
$$

Therefore, the joint PDF $p_{Z \boldsymbol{Y}}(z, x, v, t)$ of $\left(Z(t), \boldsymbol{Y}^{\mathrm{T}}(t)\right)^{\mathrm{T}}$ can be calculated using the proposed AMV-PIS by Eq.(24)(24) and (25)(25), then the PDF $p_{Z}(z, t)$ of $Z(t)$ can be obtained as a marginal distribution.

In this example, the initial values are taken as $x_{0}=0$ and $v_{0}=0$; the rate is $\lambda=3$; the linear damping coefficient is $c=0.5$; the non-linear stiffness coefficient is $\varepsilon=0.3$; the standard

设置了格式：字体颜色：蓝色

\section{设置了格式: 字体颜色：蓝色}

设置了格式: 字体颜色：蓝色

\section{设置了格式: 字体颜色: 蓝色}

设置了格式: 字体颜色：蓝色 
deviation is $\sigma=0.4$; the time step is $\Delta t=0.01$; the solving domains are $z \in[0,5]$, $x \in[-5,5]$ and $v \in[-5,5]$; the grid sizes are $\Delta z=\Delta x=\Delta v=0.04$. The PDF surfaces of $X(t)$ and $Z(t)$ against time $t \in[5,30]$ obtained by the proposed numerical method are shown in Fig.11.
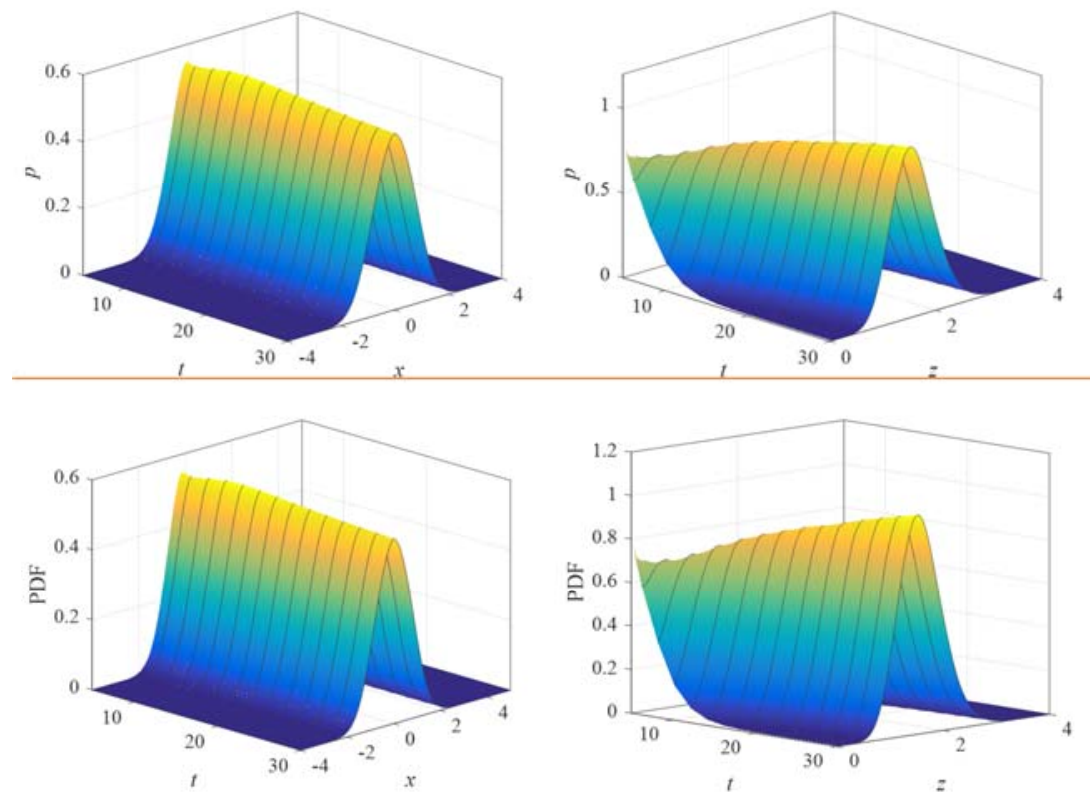

(a)

(b)

Fig.11. The PDF surfaces of $X(t)$ and $Z(t)$ of Duffing oscillator. (a) PDF surface of $X(t)$; (b) PDF surface of $Z(t)$

At the time instant $t=30$, the comparison between the numerical results using the proposed AMV-PIS and MCS of the PDF and CDF of $X(t)$ is shown in Fig.12, where performed are $10^{5}$ $10^{6}$ _samples in the MCS. 

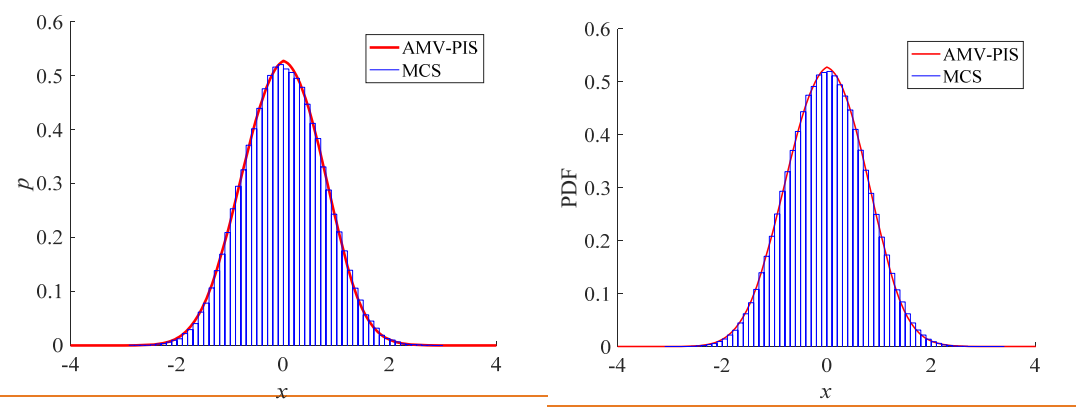

(a)
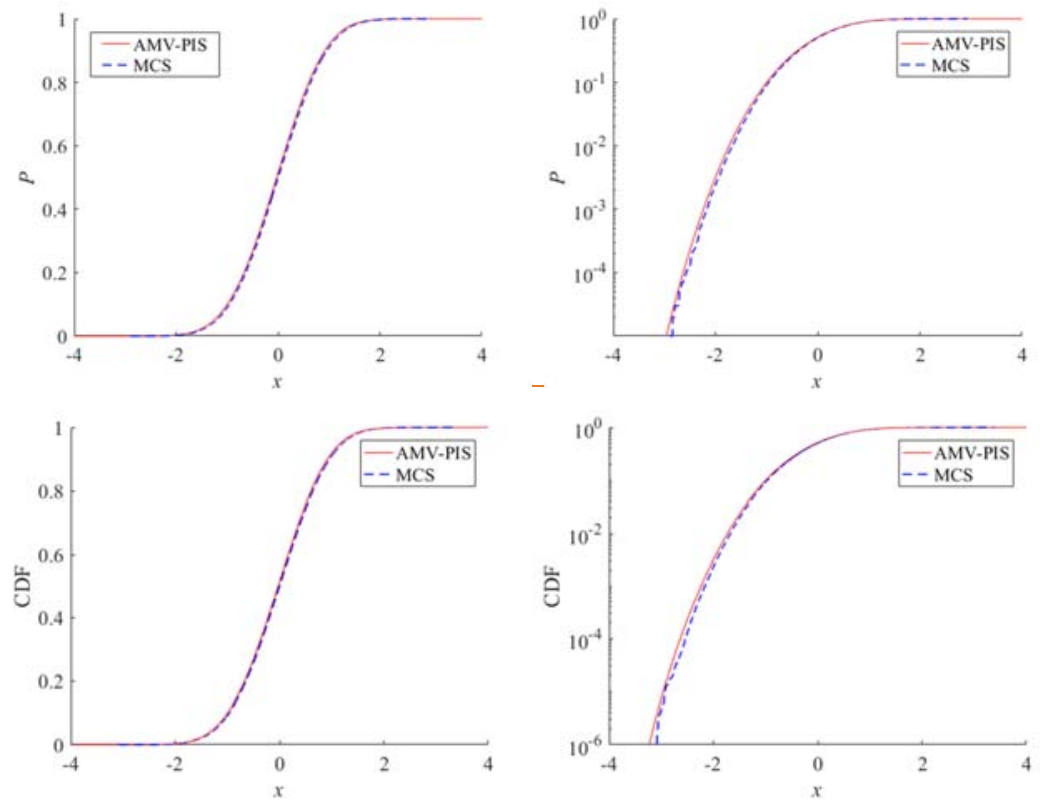

(b)

(c)

Fig.11. The comparison between AMV-PIS and MCS in terms of the PDF and CDF of the displacement response $X(t)$ of Duffing oscillator at $t=30$. (a) PDF; (b) CDF (in linear coordinates); (c) CDF (in logarithmic coordinates).

Simultaneously, the comparison between the numerical results using the AMV-PIS and MCS in terms of the PDF and CDF of $Z(t)$ is shown in Fig.12, where $10^{5} 10^{6}$ samples are performed in the MCS as well. Again, fairly good agreement is observed. 

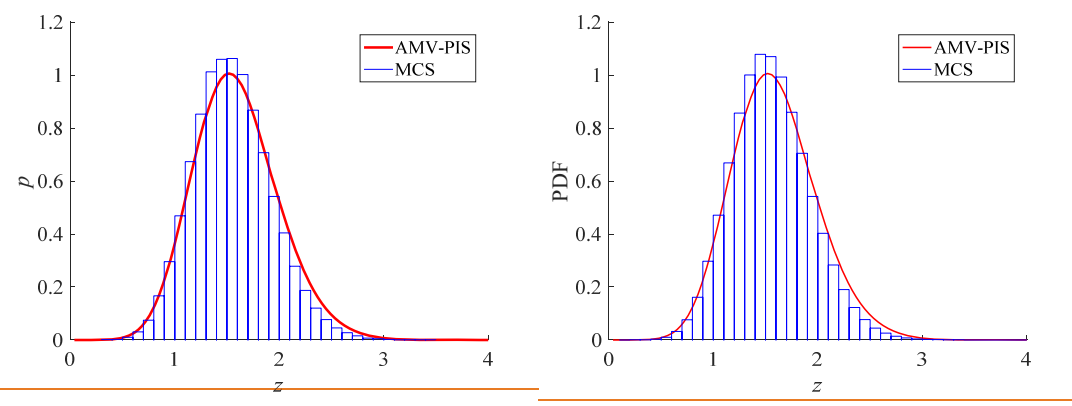

(a)
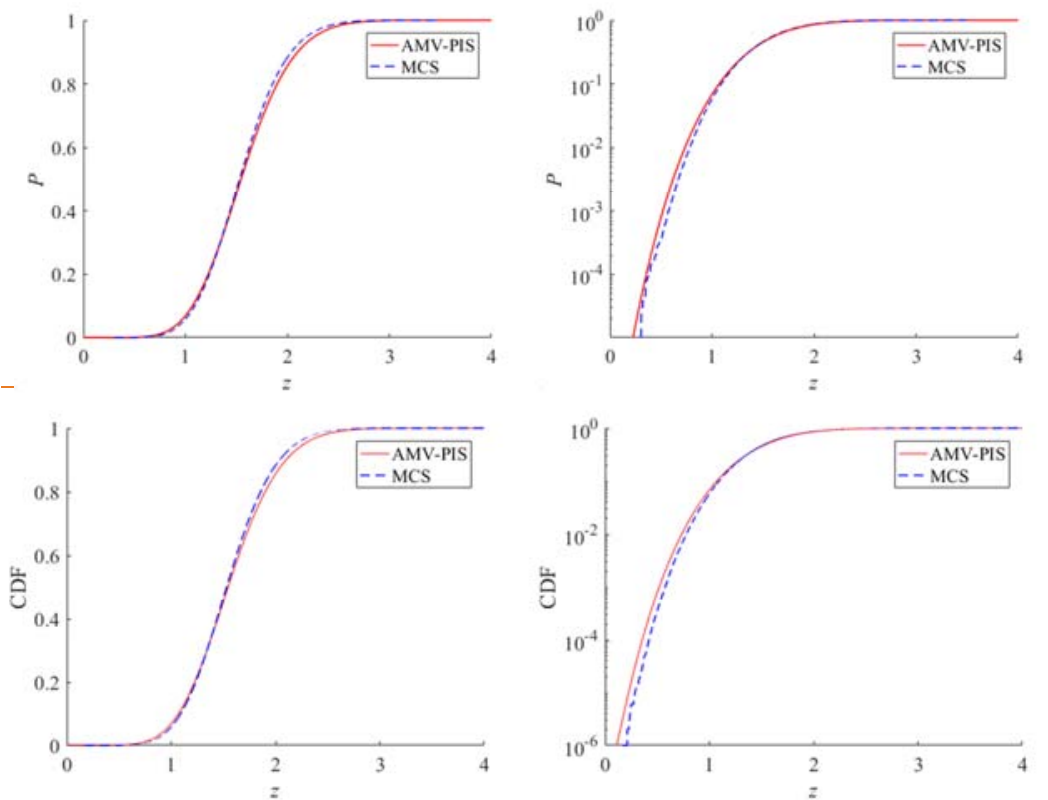

(b)

(c)

Fig.12. The comparison between AMV-PIS and MCS in terms of the PDF and CDF of the maximum value process $Z(t)$ of Duffing oscillator at $t=30$. (a) PDF; (b) CDF (in linear coordinates); (c) CDF (in logarithmic coordinates).

It can be seen from the results of the above four examples that the results of the proposed AMVPIS method are in good agreement with the results of MCS, which demonstrates convincingly that using the proposed method to obtain the PDF of time-variant extreme value process of Markov processes is effective and promising. 
Furthermore, to elarifycapture the visualized properties of samples of response enforced by Poisson white noise, two samples of compound Poisson excitation and underlying response of Duffing oscillator are shown in Fig.13. It can be seen from the figure that compound Poisson process (described by Eq.(4)) is sparse with jumped shorter or longer steps, which means that the Poisson white noise process is consisting of discrete spikes (described by Eq.(1)). This is significantly different from the Gaussian white noise excitation. Therefore, the response of a dynamical system enforced by Poisson excitation has also considerably different statistic property from the Gaussian excited systems.

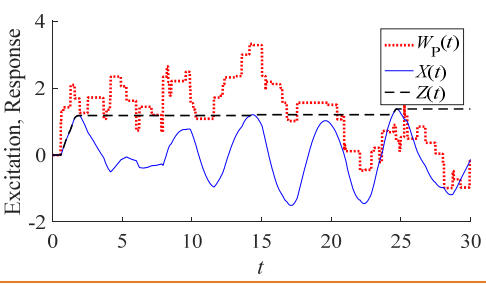

(a)

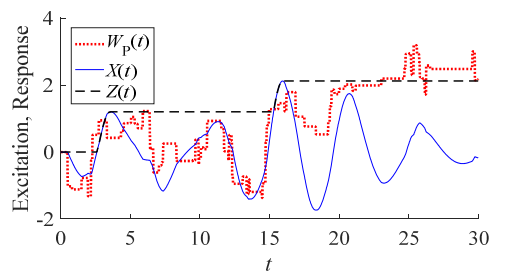

(b)
Fig.13. Two samples of compound Poisson excitation $W_{\mathrm{P}}(t)$ and underlying response $X(t)$ and $Z(t)$ of the Duffing oscillator. (a) The $1^{\text {st }}$ sample; (b) The $2^{\text {nd }}$ sample.

It can be seen from Fig. 13 that the Poisson white noise excitation is sparse and significantly different from the Gaussian excitation. Therefore, the response of a dynamic system enforced by Poisson excitation has different statistic property.

\section{Concluding remarks}

In the present paper, the augmented Markov vector process incorporated with the path integral solution for capturing the PDF of the time-variant extreme value process is further extended to the stochastic dynamical system enforced by Poisson white noise excitation $n_{5,}$ and itsThe theoretical basis and numerical implementation are elaborated in detail. In this method, the time-variant extreme value process is defined as the stochastic process whose value at a time instant is the extreme value (e.g. maximum value, minimum value, or absolute maximum value, etc.) of the underlying stochastic process during the interval from the initial time to the present time. An
设置了格式：字体：非加粗

\section{域代码已更改 \\ 域代码已更改 \\ 域代码已更改}

带格式的：缩进：首行缩进：2 字符

设置了格式：字体颜色：蓝色 
augmented Markov vector process is then constructed by combining the time-variant extreme value process and the underlying Markov process. The numerical algorithm, AMV-PIS, is then proposed based on the Chapman-Kolmogorov equation. Several examples, including linear and non-linear, one-dimensional and multi-dimensional systems, are illustrated to obtain the probability density of the time-variant extreme value process. The conclusions include:

(1) For different impulses of Poisson white noise excitation, the PDF of the time-variant extreme process can be obtained numerically by the proposed method, which is applicable to general linear or nonlinear, one-dimensional or multi-dimensional stochastic dynamic systems.

(2) The numerical results of this method are of highhave ideal accuracy. Even the calculation

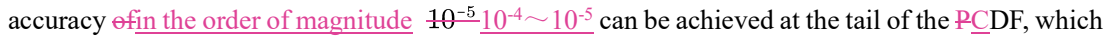
is in good agreement with the MCS results. It is of great significancet for the ealculation-evaluation of engineering reliability.

(3) For low-dimensional stochastic dynamical systems, this method has ideal computational efficiency. Due to the sparsity of the TPD of the augmented vector process, programming can be processed to have higher numerical storage and computational efficiency.

The basic idea in the present paper can be extended to more complex systems and is also possible, in principle, for non-Markov processes. However, extensive further studies are still needed in the future.

\section{Acknowledgements}

Financial supports from the National Natural Science Foundation of China (NSFC Grant Nos. 11672209, 51538010 and the National Science Fund for Distinguished Young Scholars with Grant No.51725804), the NSFC-DFG joint program (11761131014) and the International Joint Research Program of Shanghai Municipal Government (Grant No. 18160712800) are highly appreciated. 


\section{References}

[1] Tung CC. Random response of highway bridges to vehicle loads. Journal of the Engineering Mechanics Division. 1967; 93 (5): 79-94.

[2] Gao RF, Li J. Simulation of compound Poisson process based on stochastic harmonic function. Journal of Tongji University (Naturale Science). 2017; 45 (12): 1731-1738 (in Chinese).

[3] Roberts JB. The response of linear vibratory systems to random impulses. Journal of Sound and Vibration. 1965; 2 (4): 375-390.

[4] Liepmann HW. On the application of statistical concepts to the buffeting problem. Journal of the Aeronautical Sciences. 1952; 19 (12): 793-800.

[5] Lin YK. Application of nonstationary shot noise in the study of system response to a class of nonstationary excitations. ASME - Journal of Applied Mechanics. 1963; 30 (4): 555-558.

[6] Iwankiewicz R, Nielsen SRK. Solution techniques for pulse problems in non-linear stochastic dynamics. Probabilistic Engineering Mechanics. 2000; 15 (1): 25-36.

[6][7] Xu Y, Li H, Wang HY, Jia WT, Yue XL, Kurths J. The estimates of the mean first exit time of a bi-stable system excited by Poisson white noise. Journal of Applied Mechanics. 2017; 84 (9): 091004

[7][8] Wojtkiewicz SF, Johnson EA, Bergman LA, Grigoriu M, Spencer Jr BF. Response of stochastic dynamical systems driven by additive Gaussian and Poisson white noise: Solution of a forward generalized Kolmogorov equation by a spectral finite difference method. Computer Methods in Applied Mechanics \& Engineering. 1999; 168 (1-4): 73-89.

[8][9] Zeng Y, Zhu WQ. Stochastic averaging of quasi-linear systems driven by Poisson white noise. Probabilistic Engineering Mechanics. 2010; 25 (1): 99-107.

[9][10] Di Matteo A, Di Paola M, Pirrotta A. Poisson white noise parametric input and response by using complex fractional moments. Probabilistic Engineering Mechanics. 2014; 38: 119126.

[10][11] Han Q, Xu W, Yue XL, Zhang Y. First-passage time statistics in a bistable system subject to Poisson white noise by the generalized cell mapping method. Communications in Nonlinear Science and Numerical Simulation. 2015; 23 (1-3): 220-228.

[11][12] Köylüoğlu HU, Nielsen SRK, Iwankiewicz R. Response and reliability of Poisson-driven systems by path integration. Journal of Engineering Mechanics. 1995; 121 (1): 117-130.

设置了格式：字体颜色：蓝色

设置了格式：字体颜色：蓝色 
[12]13] Di Paola M, Santoro R. Non-linear systems under Poisson white noise handled by path integral solution. Journal of Vibration and Control. 2008; 14 (1-2): 35-49.

[14] Di Matteo A, Di Paola M, Pirrotta A. Path integral solution for nonlinear systems under parametric Poissonian white noise input. Probabilistic Engineering Mechanics. 2016; 44: 8998.

[13] Xu Y, Zan WR, Jia WT, Kurths J. Path integral solutions of the governing equation of SDEs excited by Lévy white noise. Journal of Computational Physics. 2019; 394 (1): 41-55.

$[15]$

[14][16] Redner S. A Guide to First-Passage Processes. Cambridge: Cambridge University Press; 2001.

[15]17] Chen JB, Li J. Dynamic response and reliability analysis of non-linear stochastic structures. Probabilistic Engineering Mechanics. 2005; 20 (1): 33-44.

[16][18] Sun JJ, Xu W, Lin ZF. Research on the reliability of friction system under combined additive and multiplicative random excitations. Communications in Nonlinear Science and Numerical Simulation. 2018; 54: 1-12.

[17][19] Melchers RE, Beck AT. Structural Reliability Analysis and Prediction. 3rd ed. Chichester: John Wiley \& Sons; 2018.

[18][20] Fisher RA, Tippett LHC. Limiting forms of the frequency distribution of the largest and smallest member of a sample. Mathematical Proceedings of the Cambridge Philosophical Society. 1928; 24 (2): 180-190

[19][21] Gumbel EJ. Statistics of Extremes. New York: Columbia University Press; 1958.

[20][22] Ang AHS, Tang WH. Probability Concepts in Engineering Planning and Design. Vol 2. New York: John Wiley and Sons; 1984.

[21][23] Newland DE. An Introduction to Random Vibration, Spectral Analysis \& Wavelet Analysis. 3rd ed. London \& New York: Longman; 1993.

[22][24] Finkenstädt B, Rootzén H. Extreme Values in Finance, Telecommunications, and the Environment. Boca Raton: Chapman and Hall/CRC; 2004.

[23][25] Naess A, Moan T. Stochastic Dynamics of Marine Structures. Cambridge: Cambridge University Press; 2013.

[24][26] Rice SO. Mathematical analysis of random noise. Bell Labs Technical Journal. 1944; 23: 30 
$282-332$.

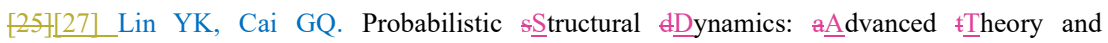
aApplication. McGraw-Hill College; 1995.

[26][28] Li J, Chen JB. Stochastic Dynamics of Structure. Singapore: John Wiley \& Sons (Asia) Pte Ltd; 2009.

[27][29] Li J, Chen JB. The principle of preservation of probability and the generalized density evolution equation. Structural Safety. 2008; 30: 65-77.

[28][30] Chen JB, Li J. The extreme value distribution and dynamic reliability analysis of nonlinear structures with uncertain parameters. Structural Safety. 2007: 29: 77-93.

[29][31] Li J, Chen JB, Fan WL. The equivalent extreme-value event and evaluation of the structural system reliability. Structural Safety. 2007; 29: 112-131.

[30][32] Mannella R, Palleschi V. Fast and precise algorithm for computer simulation of stochastic differential equations. Physical Review A. 1989; 40 (6): 3381-3386.

[31] 33] Honeycutt RL. Stochastic Runge-Kutta algorithms. I. White noise. Physical Review A. 1992; 45 (2): 600-603.

[32][34] Honeycutt RL. Stochastic Runge-Kutta algorithms. II. Colored noise. Physical Review A. 1992; 45 (2): 604-610.

[33][35] Higham DJ. An algorithmic introduction to numerical simulation of stochastic differential equations. Society for Industrial and Applied MathematicsSIAM Review,- 2001; 43 (3): 525546.

[34][36] Chen JB, Lyu MZ. A new approach for the time-variant probability density function of the maximum value of a Markov process. Physica A, 20198 (

[35][37] Snyder DL. Random p point p processes. New York: Springer-Verlag; 1975.

[36][38] Klebaner FC. Introduction to Stochastic Calculus with Application. 2nd ed. London: Imperial College Press; 2005.

[37][39] Dudley RM. Real Analysis and Probability. Cambridge: The Press Syndicate of the University of Cambridge; 1989.

[38][40] Monili A, Talkner P, Katul GG, Porporato A. First passage time statistics of Brownian motion with purely time dependent drift and diffusion. Physica A. 2011; 390: 1841-1852.

[39][41] Kou S, Zhong H. First-passage times of two-dimensional Brownian motion. Advanced 
Applied Probability. 2016; 48: 1045-1060.

[40][42] Zhu WQ. Nonlinear Stochastic Dynamics and Control - Hamiltonian Formulation. Beijing:

Science Press; 2003 (in Chinese). 